\title{
The hyperplanes of the $U_{4}(3)$ near hexagon
}

\author{
Bart De Bruyn and Sergey Shpectorov
}

February 18, 2010

\begin{abstract}
Combining theoretical arguments with calculations in the computer algebra package GAP, we are able to show that there are 27 isomorphism classes of hyperplanes in the near hexagon for the group $U_{4}(3)$. We give an explicit construction of a representative of each class and we list several combinatorial properties of such a representative.
\end{abstract}

Keywords: near hexagon, hyperplane, dual polar space, universal embedding, minimal full polarized embedding

MSC2000: 05E20, 51A45, 51A50, 51E12

\section{Introduction}

\subsection{Basic definitions}

In this subsection, we recall the basic notions regarding hyperplanes, embeddings and near polygons which are necessary to understand the results discussed in Subsection 1.2. Readers familiar with these notions might skip this subsection and go immediately to Subsection 1.2.

Let $\Gamma=(\mathcal{P}, \mathcal{L}, \mathrm{I})$ be a point-line geometry with point-set $\mathcal{P}$, line-set $\mathcal{L}$ and incidence relation $\mathrm{I} \subseteq \mathcal{P} \times \mathcal{L}$. A set $S$ of points of $\Gamma$ is called a subspace if every line containing at least two points of $S$ is completely contained in $S$. A hyperplane of $\Gamma$ is a proper subspace of $\Gamma$ which meets every line.

A full (projective) embedding of $\Gamma$ into a projective space $\Sigma$ is an injective mapping $e$ from $\mathcal{P}$ to the point-set of $\Sigma$ satisfying: (i) $\langle e(\mathcal{P})\rangle_{\Sigma}=\Sigma$; (ii) $e(L):=\{e(x) \mid x \in L\}$ is a line of $\Sigma$ for every line $L$ of $\Gamma$. The dimensions $\operatorname{dim}(\Sigma)$ and $\operatorname{dim}(\Sigma)+1$ are respectively called the projective dimension and the vector dimension of $e$. If $e: \Gamma \rightarrow \Sigma$ is a full embedding of $\Gamma$, then for every hyperplane $\alpha$ of $\Sigma, e^{-1}(\alpha \cap e(\mathcal{P}))$ is a hyperplane of $\Gamma$. We say that the hyperplane $e^{-1}(\alpha \cap e(\mathcal{P}))$ of $\Gamma$ arises from the embedding e.

Two full embeddings $e_{1}: \Gamma \rightarrow \Sigma_{1}$ and $e_{2}: \Gamma \rightarrow \Sigma_{2}$ of $\Gamma$ are called isomorphic $\left(e_{1} \cong e_{2}\right)$ if there exists an isomorphism $f: \Sigma_{1} \rightarrow \Sigma_{2}$ such that $e_{2}=f \circ e_{1}$. If $e: \Gamma \rightarrow \Sigma$ is a full embedding of $\Gamma$ and if $U$ is a subspace of $\Sigma$ satisfying 
(C1) $\langle U, e(p)\rangle_{\Sigma} \neq U$ for every point $p$ of $\Gamma$

(C2) $\left\langle U, e\left(p_{1}\right)\right\rangle_{\Sigma} \neq\left\langle U, e\left(p_{2}\right)\right\rangle_{\Sigma}$ for any two distinct points $p_{1}$ and $p_{2}$ of $\Gamma$,

then there exists a full embedding $e / U$ of $\Gamma$ into the quotient space $\Sigma / U$ mapping each point $p$ of $\Gamma$ to $\langle U, e(p)\rangle_{\Sigma}$. If $e_{1}: \Gamma \rightarrow \Sigma_{1}$ and $e_{2}: \Gamma \rightarrow \Sigma_{2}$ are two full embeddings of $\Gamma$, then we say that $e_{1} \geq e_{2}$ if there exists a subspace $U$ in $\Sigma_{1}$ satisfying $(\mathrm{C} 1),(\mathrm{C} 2)$ and $e_{1} / U \cong e_{2}$. If $e: \Gamma \rightarrow \Sigma$ is a full embedding of $\Gamma$, then by Ronan [28], there exists (up to isomorphism) a unique full embedding $\widetilde{e}: \Gamma \rightarrow \widetilde{\Sigma}$ satisfying (i) $\widetilde{e} \geq e$, (ii) if $e^{\prime} \geq e$ for some full embedding $e^{\prime}$ of $\Gamma$, then $\widetilde{e} \geq e^{\prime}$. We say that $\widetilde{e}$ is universal relative to $e$. If $\widetilde{e} \cong e$ for some full embedding $e$ of $\Gamma$, then we say that $e$ is relatively universal. A full embedding $e$ of $\Gamma$ is called absolutely universal if it is universal relative to any full embedding of $\Gamma$ defined over the same division ring as $e$.

Suppose $\Gamma=(\mathcal{P}, \mathcal{L}, \mathrm{I})$ is a point-line geometry with three points on each line. If $X_{1}$ and $X_{2}$ are two sets of points of $\Gamma$, then we define $X_{1} * X_{2}=\left(X_{1} \cap X_{2}\right) \cup\left(\mathcal{P} \backslash\left(X_{1} \cup X_{2}\right)\right)$. Clearly, $X_{1} * \mathcal{P}=X_{1}, X_{1} * X_{1}=\mathcal{P}, X_{1} * X_{2}=X_{2} * X_{1}$ and $X_{1} *\left(X_{2} * X_{3}\right)=\left(X_{1} * X_{2}\right) * X_{3}$ for all $X_{1}, X_{2}, X_{3} \subseteq \mathcal{P}$. If $X_{1}$ and $X_{2}$ are two distinct hyperplanes of $\Gamma$, then $X_{1} * X_{2}$ is again a hyperplane of $\Gamma$. If $\mathcal{H}(\Gamma)$ denotes the set of all hyperplanes of $\Gamma$, then $\mathcal{H}(\Gamma) \cup\{\mathcal{P}\}$ carries the structure of a vector space over the field $\mathbb{F}_{2}$ if we put $H_{1}+H_{2}:=H_{1} * H_{2}, 0 \cdot H_{1}:=\mathcal{P}$ and $1 \cdot H_{1}:=H_{1}$ for all $H_{1}, H_{2} \in \mathcal{H}(\Gamma)$.

Suppose $\Gamma=(\mathcal{P}, \mathcal{L}, \mathrm{I})$ is a fully embeddable point-line geometry with three points on each line. Then by Ronan [28], $\Gamma$ admits the absolutely universal embedding and every hyperplane of $\Gamma$ arises from this embedding. We now give a description of the absolutely universal embedding of $\Gamma$. Let $V$ be a vector space over the field $\mathbb{F}_{2}$ with a basis $B$ whose vectors are indexed by the elements of $\mathcal{P}$, say $B=\left\{\bar{v}_{p} \mid p \in \mathcal{P}\right\}$. Let $W$ denote the subspace of $V$ generated by all vectors $\bar{v}_{p_{1}}+\bar{v}_{p_{2}}+\bar{v}_{p_{3}}$ where $\left\{p_{1}, p_{2}, p_{3}\right\}$ is a line of $\Gamma$. Then the map $p \in \mathcal{P} \mapsto\left\{\bar{v}_{p}+W, W\right\}$ defines a full embedding of $\Gamma$ into the projective space $\mathrm{PG}(V / W)$ which is isomorphic to the absolutely universal embedding of $\Gamma$.

A near polygon is a partial linear space $\Gamma=(\mathcal{P}, \mathcal{L}, \mathrm{I})$ with the property that for every point $p \in \mathcal{P}$ and every line $L \in \mathcal{L}$, there exists a unique point on $L$ nearest to $p$. Here, distances $\mathrm{d}(\cdot, \cdot)$ are measured in the collinearity graph $G$ of $\Gamma$. If $d$ is the diameter of $G$, then the near polygon is called a near $2 d$-gon. We will always assume that $d$ is finite. The near 0 -gon consists of one point (no lines) and the near 2-gons are precisely the lines. Near quadrangles are usually called generalized quadrangles (GQ's).

For every point $x$ of a near polygon $\Gamma=(\mathcal{P}, \mathcal{L}, \mathrm{I})$, for every non-empty subset $X$ of $\mathcal{P}$ and every $i \in \mathbb{N}$, we define $\Gamma_{i}(x):=\{y \in \mathcal{P} \mid \mathrm{d}(x, y)=i\}, x^{\perp}=\Gamma_{0}(x) \cup \Gamma_{1}(x), \mathrm{d}(x, X)=$ $\min \{\mathrm{d}(x, y) \mid y \in X\}, \Gamma_{i}(X)=\{y \in \mathcal{P} \mid \mathrm{d}(y, X)=i\}$. If $X_{1}$ and $X_{2}$ are two non-empty sets of points of $\Gamma$, then we define $\mathrm{d}\left(X_{1}, X_{2}\right):=\min \left\{\mathrm{d}\left(x_{1}, x_{2}\right) \mid x_{1} \in X_{1}\right.$ and $\left.x_{2} \in X_{2}\right\}$.

A near polygon is called dense if every line is incident with at least three points and if every two points at distance 2 have at least two common neighbours. By Theorem 4 of Brouwer and Wilbrink [5], every two points of a dense near polygon at distance $\delta$ from each other are contained in a unique convex sub near $2 \delta$-gon. These convex sub near $2 \delta$-gons are called quads 
if $\delta=2$. If $X_{1}, X_{2}, \ldots, X_{k}$ are $k$ (sets of) points of a near polygon $\Gamma$, then $\left\langle X_{1}, X_{2}, \ldots, X_{k}\right\rangle$ denotes the smallest convex subspace of $\Gamma$ containing $X_{1}, X_{2}, \ldots, X_{k}$.

The set of points at non-maximal distance from a given point $x$ of a dense near polygon $\Gamma$ is a hyperplane of $\Gamma$. We call this hyperplane the singular hyperplane with deepest point $x$ and we denote it by $H_{x}$. By Shult [30, Lemma 6.1], every hyperplane of a dense near polygon is also a maximal subspace. If $H$ is a hyperplane of a dense near polygon $\Gamma$ and if $Q$ is a quad of $\Gamma$, then either $Q \subseteq H$ or $Q \cap H$ is a hyperplane of $Q$. By Payne and Thas [26, 2.3.1], one of the following cases then occurs:

(i) $Q \subseteq H$;

(ii) $Q \cap H=x^{\perp} \cap Q$ for some point $x$ of $Q$;

(iii) $Q \cap H$ is a proper subquadrangle of $Q$;

(iv) $Q \cap H$ is an ovoid of $Q$, i.e. a set of points of $Q$ meeting each line in a unique point. If case (i), (ii), (iii), respectively (iv), occurs, then $Q$ is called deep, singular, subquadrangular, respectively ovoidal, with respect to $H$.

Let $e: \Gamma \rightarrow \Sigma$ be a full embedding of a dense near polygon $\Gamma=(\mathcal{P}, \mathcal{L}, \mathrm{I})$. If $H$ is a hyperplane of $\Gamma$ arising from $e$, then since $H$ is a maximal subspace of $\Gamma,\langle e(H)\rangle_{\Sigma}$ is a hyperplane of $\Sigma$ and $\langle e(H)\rangle_{\Sigma} \cap e(\mathcal{P})=e(H)$. The embedding $e$ is called polarized if every singular hyperplane of $\Gamma$ arises from $e$. If $e$ is polarized, then the subspace $R_{e}:=\bigcap_{x \in \mathcal{P}}\left\langle e\left(H_{x}\right)\right\rangle_{\Sigma}$ satisfies the conditions (C1) and (C2) mentioned above and $e / R_{e}: \Gamma \rightarrow \Sigma / R_{e}$ is also a full polarized embedding of $\Gamma$, see [15]. The embedding $e / R_{e}$ is called a minimal full polarized embedding of $\Gamma$.

Now, let $\Gamma$ be a dense near polygon with three points on each line. Then $\Gamma$ has a full embedding, see e.g. [15], and hence admits the absolutely universal embedding $\widetilde{e}: \Gamma \rightarrow \widetilde{\Sigma}$ by Ronan [28]. The embedding $\bar{e}:=\widetilde{e} / R_{\tilde{e}}$ is the unique minimal full polarized embedding of $\Gamma$ : we have $e^{\prime} \geq \bar{e}$ for any full polarized embedding $e^{\prime}$ of $\Gamma$. By Ronan [28], every hyperplane of $\Gamma$ arises from $\widetilde{e}$. The set $\mathcal{H}(\Gamma)$ of all hyperplanes of $\Gamma$ carries the structure of a projective space isomorphic to (the dual of) $\widetilde{\Sigma}$ if one takes the sets $\left\{H_{1}, H_{2}, H_{1} * H_{2}\right\}, H_{1}, H_{2} \in \mathcal{H}(\Gamma)$ with $H_{1} \neq H_{2}$, as lines. Let $\mathcal{H}^{\prime}(\Gamma)$ denote the subspace of $\mathcal{H}(\Gamma)$ generated by all singular hyperplanes. Then $\mathcal{H}^{\prime}(\Gamma)$ coincides with the set of all hyperplanes arising from the minimal full polarized embedding $\bar{e}$.

Remark. If $\Gamma$ is a dense near polygon with three points per line, then the minimal full polarized embedding $\bar{e}$ is sometimes also called the near polygon embedding.

\subsection{The results}

Classification results for hyperplanes have been obtained for several classes of dense near polygons with three points per line, see [4] for the $M_{24}$ near hexagon $\mathbb{E}_{2}$, [27] (see also [14, Section 9]) for the dual polar space $D W(5,2),[16,17]$ for the dual polar space $D H(5,4)$ and [18] for the near hexagon $\mathbb{H}_{3}$ on the 2 -factors of the complete graph $K_{8}$. The aim of the present paper is to give a classification of the hyperplanes of another dense near hexagon with three points per line, namely the $U_{4}(3)$ near hexagon $\mathbb{E}_{3}$. There are still three dense near hexagons with three points 
per line whose hyperplane classification is still open (namely $\mathbb{E}_{1}, \mathbb{G}_{3}$ and $Q(5,2) \otimes Q(5,2)$ ). In a sequel paper [19] we will deal with the classification of the hyperplanes of the near hexagon $\mathbb{E}_{1}$ which is related to the extended ternary Golay code. We start by giving a model for the near hexagon under consideration in the present paper.

Consider in $\mathrm{PG}(6,3)$ a nonsingular parabolic quadric $Q(6,3)$ and a non-tangent hyperplane $\pi$ intersecting $Q(6,3)$ in a nonsingular elliptic quadric $Q^{-}(5,3)$ of $\pi$. There is a polarity associated with $Q(6,3)$ and we call two points of $\mathrm{PG}(6,3)$ orthogonal when one of them is contained in the polar hyperplane of the other. Let $N$ denote the set of 126 internal points of $Q(6,3)$ which are contained in $\pi$, i.e. the set of all 126 points in $\pi$ for which the polar hyperplane intersects $Q(6,3)$ in a nonsingular elliptic quadric. Let $\mathbb{E}_{3}$ be the following point-line geometry:

- the points of $\mathbb{E}_{3}$ are the 6-tuples of mutually orthogonal points of $N$;

- the lines of $\mathbb{E}_{3}$ are the pairs of mutually orthogonal points of $N$;

- incidence is reverse containment.

The incidence structure $\mathbb{E}_{3}$ is a dense near hexagon with three points on each line. The above description of the near hexagon has been taken from Brouwer \& Wilbrink [5] and Brouwer et al. [3]. Other descriptions of this near hexagon can be found in Aschbacher [1, p. 31], De Bruyn [13], Kantor [23, p. 240], Pasini \& Shpectorov [25, p. 279], and Ronan \& Smith [29, p. 285]. The automorphism group $A u t\left(\mathbb{E}_{3}\right)$ of $\mathbb{E}_{3}$ is isomorphic to $P O_{6}^{-}(3)$ and contains $U_{4}(3)$ as a subgroup of index 4 . Every quad of $\mathbb{E}_{3}$ is isomorphic to either $W(2)$ or $Q(5,2)$. Here, $W(2)$ denotes the unique GQ with three points per line and three lines through each point, and $Q(5,2)$ denotes the unique GQ with three points per line and 5 lines through each point.

The aim of this paper is to enumerate the isomorphism classes of hyperplanes of the near hexagon $\mathbb{E}_{3}$, to give at least one explicit construction of a representative of each isomorphism class and to list several combinatorial properties of such a representative. For some isomorphism classes, we will indeed give more than one construction for a representative, thus obtaining distinct constructions for the same hyperplane. The following is the main result of this paper.

Main Theorem. The $U_{4}(3)$ near hexagon $\mathbb{E}_{3}$ has 27 isomorphism classes of hyperplanes. Among these 27 isomorphism classes, there are 17 classes whose hyperplanes arise from the minimal full polarized embedding of $\mathbb{E}_{3}$.

By Ronan [28], every hyperplane of $\mathbb{E}_{3}$ arises from the absolutely universal embedding of $\mathbb{E}_{3}$. Yoshiara [31] showed that this absolutely universal embedding has vector dimension 21, see also Bardoe [2] or De Bruyn [10] for alternative proofs. This implies that $\mathbb{E}_{3}$ has precisely $\left|\mathcal{H}\left(\mathbb{E}_{3}\right)\right|=2^{21}-1=2097151$ hyperplanes. We will show in Section 2 (Proposition 2.1) that the minimal full polarized embedding of $\mathbb{E}_{3}$ has vector dimension 20 . This implies that $\mathcal{H}^{\prime}\left(\mathbb{E}_{3}\right)$ is a hyperplane of $\mathcal{H}\left(\mathbb{E}_{3}\right)$. So, $\mathcal{H}^{\prime}\left(\mathbb{E}_{3}\right)$ is the subspace of $\mathcal{H}\left(\mathbb{E}_{3}\right)$ generated by all singular hyperplanes of $\mathbb{E}_{3}$, and the whole space $\mathcal{H}\left(\mathbb{E}_{3}\right)$ is generated by all singular hyperplanes + one extra hyperplane of $\mathcal{H}\left(\mathbb{E}_{3}\right) \backslash \mathcal{H}^{\prime}\left(\mathbb{E}_{3}\right)$. We will call the hyperplanes of $\mathcal{H}^{\prime}\left(\mathbb{E}_{3}\right)$ the Type $A$ hyperplanes and the hyperplanes of $\mathcal{H}\left(\mathbb{E}_{3}\right) \backslash \mathcal{H}^{\prime}\left(\mathbb{E}_{3}\right)$ the Type $B$ hyperplanes. 


\begin{tabular}{|c||c|c|c|c|c|c|c|c|c|c|c|c|c|c|c|c|c|}
\hline & A1 & A2 & A3 & A4 & A5 & A6 & A7 & A8 & A9 & A10 & A11 & A12 & A13 & A14 & A15 & A16 & A17 \\
\hline \hline A1 & 30 & - & 96 & - & 120 & 320 & - & - & - & - & - & - & - & - & - & - & - \\
\hline A2 & - & - & - & - & 15 & - & 360 & 192 & - & - & - & - & - & - & - & - & - \\
\hline A3 & 12 & - & 15 & - & - & 240 & 180 & - & 90 & 30 & - & - & - & - & - & - & - \\
\hline A4 & - & - & - & 21 & - & - & - & - & - & - & 210 & 336 & - & - & - & - & - \\
\hline A5 & 8 & 1 & - & - & 6 & 192 & 72 & - & 96 & - & - & - & 64 & 128 & - & - & - \\
\hline A6 & 2 & - & 12 & - & 18 & 87 & 108 & - & 72 & 12 & - & - & 12 & 72 & 36 & 1 & 135 \\
\hline A7 & - & 1 & 4 & - & 3 & 48 & 131 & 8 & 52 & - & 8 & 64 & 8 & 80 & 64 & - & 96 \\
\hline A8 & - & 12 & - & - & - & - & 180 & 45 & - & - & - & - & 30 & 120 & 180 & - & - \\
\hline A9 & - & - & 4 & - & 8 & 64 & 104 & - & 53 & 22 & 8 & 48 & - & 96 & - & - & 160 \\
\hline A10 & - & - & 12 & - & - & 96 & - & - & 198 & 53 & - & 48 & - & - & - & 16 & 144 \\
\hline A11 & - & - & - & 6 & - & - & 144 & - & 72 & - & 9 & 240 & - & 96 & - & - & - \\
\hline A12 & - & - & - & 1 & - & - & 120 & - & 45 & 5 & 25 & 91 & - & 100 & 60 & - & 120 \\
\hline A13 & - & - & - & - & 28 & 56 & 84 & 14 & - & - & - & - & 21 & 168 & 28 & - & 168 \\
\hline A14 & - & - & - & - & 6 & 36 & 90 & 6 & 54 & - & 6 & 60 & 18 & 123 & 60 & - & 108 \\
\hline A15 & - & - & - & - & - & 36 & 144 & 18 & - & - & - & 72 & 6 & 120 & 63 & - & 108 \\
\hline A16 & - & - & - & - & - & 108 & - & - & - & 216 & - & - & - & - & - & - & 243 \\
\hline A17 & - & - & - & - & - & 60 & 96 & - & 80 & 8 & - & 64 & 16 & 96 & 48 & 1 & 98 \\
\hline
\end{tabular}

Table 1: The "action" of the singular hyperplanes on a Type A hyperplane

In the computer algebra package GAP $([22])$, we have implemented the absolutely universal embedding space $\widetilde{\Sigma}$ of $\mathbb{E}_{3}$ and its dual space $\widetilde{\Sigma}^{*}$, together with the action of $A u t\left(\mathbb{E}_{3}\right)$ on these spaces. Notice that there exists a natural bijective correspondence between the points of $\widetilde{\Sigma}^{*}$ and the hyperplanes of $\mathbb{E}_{3}$. We have randomly chosen points in the dual space $\widetilde{\Sigma}^{*}$ and calculated their orbits. We have repeated this process till the union of all orbits did coincide with the whole point set of $\widetilde{\Sigma}^{*}$. We found that there are 27 distinct orbits, i.e. 27 distinct isomorphism classes of hyperplanes of $\mathbb{E}_{3}$.

To obtain all isomorphism classes of Type A hyperplanes one can also proceed as follows. Start with a given singular hyperplane $H$. The hyperplane orbit containing $H$ has size 567 (= the total number of points of $\mathbb{E}_{3}$ ). Construct all hyperplanes of the form $H * H_{x}$, where $x$ ranges over all points of $\mathbb{E}_{3}$. For each new hyperplane $H^{\prime}$ arising, calculate the size of its orbit, construct all hyperplanes of the form $H^{\prime} * H_{x}$ and determine once again the sizes of the orbits of the new hyperplanes which arise. Repeat this until the sum of the sizes of all found hyperplane orbits equals $\left|\mathcal{H}^{\prime}\left(\mathbb{E}_{3}\right)\right|=2^{20}-1$. A priori, it might be possible that we need 20 singular hyperplanes to generate all Type A hyperplanes. It turns out however that at most 5 singular hyperplanes suffice. The results of this method for constructing the isomorphism classes of Type A hyperplanes are listed in Table 1. There are 17 orbits of Type A hyperplanes which we label by A1, A2, .., A17. In Sections 3 and 5, we will give an explicit construction of a representative of each orbit. We have labeled the hyperplane orbits in accordance with the order in which we will describe these representatives. The Type A1 hyperplanes are precisely the singular hyperplanes. The number 192 in entry (A5,A6) of Table 1 means that for a given hyperplane $H$ of Type A5, there are 192 singular hyperplanes $H_{x}$ such that $H * H_{x}$ is a hyperplane of Type A6. Notice that the sum of all entries in a given row distinct from "row A1" equals 567. The sum of the entries in "row A1" is equal to 566 since we need to combine 


\begin{tabular}{|c||c|c|c|c|c|c|c|c|c|c|}
\hline & B1 & B2 & B3 & B4 & B5 & B6 & B7 & B8 & B9 & B10 \\
\hline \hline B1 & - & 405 & 162 & - & - & - & - & - & - & - \\
\hline B2 & 1 & 80 & 6 & - & 36 & 48 & 144 & - & 72 & 180 \\
\hline B3 & 7 & 105 & 35 & - & - & - & - & - & 210 & 210 \\
\hline B4 & - & - & - & - & - & 105 & 252 & 210 & - & - \\
\hline B5 & - & 36 & - & - & 36 & 72 & 252 & 48 & 9 & 114 \\
\hline B6 & - & 8 & - & 1 & 12 & 160 & 204 & 26 & 12 & 144 \\
\hline B7 & - & 20 & - & 2 & 35 & 170 & 195 & 20 & 15 & 110 \\
\hline B8 & - & - & - & 15 & 60 & 195 & 180 & 57 & - & 60 \\
\hline B9 & - & 72 & 12 & - & 9 & 72 & 108 & - & 24 & 270 \\
\hline B10 & - & 30 & 2 & - & 19 & 144 & 132 & 8 & 45 & 187 \\
\hline
\end{tabular}

Table 2: The "action" of the singular hyperplanes on a Type B hyperplane

two distinct singular hyperplanes. Some of the Type A hyperplanes can easily be constructed without giving explicitly a generating set of singular hyperplanes. This is the case for the hyperplanes of Type A2, A3 and A4. We call the hyperplanes of Type A1, A2, A3 and A4 the basic hyperplanes of Type $A$ and show how all the remaining hyperplanes of Type A can be constructed from them. For some hyperplane classes we will give more than one construction for a representative. The hyperplanes of Type A16 turn out to have a nice structure. In Section 6 we will give an alternative construction for these hyperplanes and discuss their structure.

A hyperplane of $\mathbb{E}_{3}$ on 405 points was constructed in [10, Section 3]. This hyperplane is isomorphic to the hyperplane of Type B on 405 points which we will describe in Proposition 3.2. We will refer to this particular hyperplane as a Type B1 hyperplane. The points and lines contained in a Type B1 hyperplane define a dense near hexagon which is isomorphic to the near hexagon $\mathbb{G}_{3}$ which we will define in Section 2. To obtain all isomorphism classes of Type B hyperplanes one can proceed in a similar way as above by starting from a given hyperplane of Type B1 and combining it with singular hyperplanes. The results of this method for constructing the isomorphism classes of Type B hyperplanes are listed in Table 2. There are 10 distinct orbits of Type B hyperplanes which we label by B1, B2, .., B10. In Section 5.8, we will give an explicit construction for a representative of each orbit. Again, we have labeled the hyperplane orbits in accordance with the order in which we will describe these representatives.

In Table 3 we list the sizes of the hyperplane orbits and several combinatorial properties of the hyperplanes. This information is obtained by means of computer computations. From Table 3 we observe the following: (i) if $H$ is a Type A hyperplane, then $8 \mid v-7$ and every point of $H$ is incident with either $3,5,7,9,11$ or 15 lines which are contained in $H$; (ii) if $H$ is a Type B hyperplane, then $16 \mid v-5$ and every point of $H$ is incident with either 4, 6, 8, 10 or 12 lines which are contained in $H$. Some of the hyperplanes of $\mathbb{E}_{3}$ carry the structure of a near hexagon. 


\begin{tabular}{|c|c|c|c|c|c|c|c|c|c|c|c|}
\hline & $N$ & $v$ & $l$ & line distribution & $\mathrm{DE}$ & $\mathrm{SU}$ & SI & $\mathrm{de}$ & $\mathrm{su}$ & si & OV \\
\hline 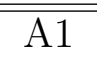 & 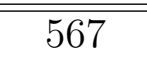 & 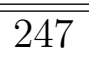 & 4435 & $3^{120} 5^{96} 15^{31}$ & $\overline{66}$ & $\overline{\overline{0}}$ & $\overline{\overline{120}}$ & 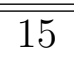 & 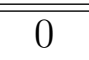 & 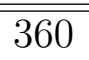 & 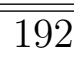 \\
\hline $\mathrm{A} 2$ & 567 & 375 & 1395 & $11^{360} 15^{15}$ & 30 & 96 & 0 & 127 & 320 & 120 & 0 \\
\hline A3 & 4536 & 327 & 1035 & $5^{12} 7^{30} 9^{180} 11^{90} 15^{15}$ & 16 & 80 & 30 & 57 & 240 & 270 & 0 \\
\hline $\mathrm{A} 4$ & 324 & 231 & 315 & $3^{210} 15^{21}$ & 0 & 0 & 126 & 21 & 0 & 210 & 336 \\
\hline A5 & 8505 & 311 & 915 & $3^{8} 7^{48} 9^{224} 11^{24} 15^{7}$ & 6 & 96 & 24 & 55 & 192 & 256 & 64 \\
\hline A6 & 90720 & 271 & 615 & $3^{18} 5^{66} 7^{120} 9^{58} 11^{9}$ & 0 & 60 & 66 & 6 & 144 & 297 & 120 \\
\hline A7 & 204120 & 279 & 675 & $3^{10} 5^{68} 7^{102} 9^{76} 11^{22} 15^{1}$ & 2 & 64 & 60 & 11 & 176 & 268 & 112 \\
\hline A8 & 9072 & 255 & 495 & $3^{30} 5^{180} 11^{45}$ & 0 & 36 & 90 & 0 & 120 & 255 & 192 \\
\hline A9 & 102060 & 295 & 795 & $3^{2} 5^{24} 7^{132} 9^{88} 11^{48} 15^{1}$ & 4 & 80 & 42 & 29 & 192 & 266 & 80 \\
\hline A10 & 11340 & 263 & 555 & $3^{18} 5^{144} 7^{48} 9^{16} 11^{36} 15^{1}$ & 0 & 48 & 78 & 9 & 96 & 318 & 144 \\
\hline A11 & 11340 & 327 & 1035 & $3^{6} 9^{240} 11^{72} 15^{9}$ & 12 & 96 & 18 & 65 & 256 & 198 & 48 \\
\hline A12 & 108864 & 271 & 615 & $3^{20} 5^{66} 7^{120} 9^{50} 11^{15}$ & 0 & 60 & 66 & 6 & 160 & 265 & 136 \\
\hline A13 & 19440 & 287 & 735 & $3^{14} 7^{168} 9^{84} 11^{21}$ & 0 & 84 & 42 & 28 & 168 & 259 & 112 \\
\hline A14 & 181440 & 279 & 675 & $3^{9} 5^{54} 7^{117} 9^{90} 11^{9}$ & 0 & 72 & 54 & 15 & 168 & 264 & 120 \\
\hline A15 & 90720 & 303 & 855 & $5^{18} 7^{108} 9^{114} 11^{63}$ & 6 & 84 & 36 & 34 & 224 & 237 & 72 \\
\hline A16 & 840 & 351 & 1215 & $9^{108} 11^{243}$ & 18 & 108 & 0 & 108 & 216 & 243 & 0 \\
\hline A17 & 204120 & 287 & 735 & $5^{56} 7^{96} 9^{116} 11^{19}$ & 2 & 76 & 48 & 20 & 184 & 267 & 96 \\
\hline$\overline{\mathrm{B} 1}$ & 112 & 2405 & 1620 & $12^{405}$ & 445 & "81 & 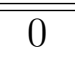 & 162 & 2405 & $\overline{0}$ & 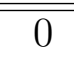 \\
\hline $\mathrm{B} 2$ & 45360 & 277 & 660 & $4^{60} 6^{48} 8^{144} 12^{25}$ & 3 & 57 & 66 & 6 & 189 & 252 & 120 \\
\hline B3 & 2592 & 245 & 420 & $4^{210} 12^{35}$ & 0 & 21 & 105 & 0 & 105 & 210 & 252 \\
\hline B4 & 2592 & 357 & 1260 & $10^{252} 12^{105}$ & 21 & 105 & 0 & 112 & 245 & 210 & 0 \\
\hline B5 & 45360 & 309 & 900 & $6^{48} 8^{144} 10^{72} 12^{45}$ & 9 & 81 & 36 & 38 & 229 & 252 & 48 \\
\hline B6 & 272160 & 293 & 780 & $4^{12} 6^{64} 8^{144} 10^{60} 12^{13}$ & 3 & 81 & 42 & 28 & 189 & 262 & 88 \\
\hline B7 & 326592 & 277 & 660 & $4^{40} 6^{80} 8^{120} 10^{32} 12^{5}$ & 1 & 65 & 60 & 10 & 165 & 280 & 112 \\
\hline B8 & 36288 & 261 & 540 & $4^{90} 6^{120} 10^{36} 12^{15}$ & 0 & 45 & 81 & 6 & 105 & 300 & 156 \\
\hline B9 & 45360 & 309 & 900 & $4^{12} 8^{192} 10^{72} 12^{33}$ & 7 & 89 & 30 & 42 & 237 & 216 & 72 \\
\hline $\mathrm{B} 10$ & 272160 & 277 & 660 & $4^{42} 6^{64} 8^{144} 10^{24} 12^{3}$ & 0 & 69 & 57 & 12 & 169 & 262 & 124 \\
\hline
\end{tabular}

The number $N$ denotes the total number of hyperplanes in the relevant orbit, $v$ and $l$ denote the number of points and lines which are contained in the hyperplane $H$. The notation $t_{1}^{n_{1}} t_{2}^{n_{2}} \cdots t_{k}^{n_{k}}$ means that there are precisely $n_{i}, i \in\{1, \ldots, k\}$, points of $H$ which are incident with precisely $t_{i}$ lines that are contained $H$. The numbers $D E, S U$, respectively $S I$, denote the number of $Q(5,2)$ quads which are deep, subquadrangular, respectively singular, with respect to $H$. (Notice that the GQ $Q(5,2)$ has no ovoids, see Payne and Thas [26, 3.4.1].) The numbers $d e, s u$, si, respectively ov, denote the number of $W(2)$-quads which are deep, subquadrangular, singular, respectively ovoidal, with respect to $H$.

Table 3: Some combinatorial properties of the hyperplanes 
Theorem 1.1 If $H$ is a hyperplane of $\mathbb{E}_{3}$ without ovoidal $W(2)$-quads, then the point-line geometry $\Gamma$ induced on $H$ is a near hexagon.

Proof. We show that $\Gamma$ is isometrically embedded into $\mathbb{E}_{3}$. Let $x_{1}$ and $x_{2}$ be two points of $\Gamma$ which lie at distance $\delta$ in $\Gamma$ and at distance $d$ in $\mathbb{E}_{3}$. Obviously, $\delta \geq d$. We need to show that $\delta=d$.

If $d \leq 1$, then $\delta=d$ since $H$ is a subspace.

Suppose $d=2$. Since the quad $\left\langle x_{1}, x_{2}\right\rangle$ is either deep, singular or subquadrangular, there exists a point in $x_{1}^{\perp} \cap x_{2}^{\perp} \cap H$. Hence, $\delta=2$.

Suppose $d=3$. Let $Q$ be an arbitrary quad through $x_{1}$. Since $Q$ is either deep, singular or subquadrangular, there exists a line $L \subseteq Q \cap H$ through $x_{1}$. This line $L$ contains a point $y$ which lies at distance 2 from $x_{2}$ in the near hexagon $\mathbb{E}_{3}$. By the previous paragraph, the distance between $y$ and $x_{2}$ in $\Gamma$ is also equal to 2 . It follows that $\delta=3$.

Since $\Gamma$ is isometrically embedded into $\mathbb{E}_{3}$ and $\mathbb{E}_{3}$ is a near polygon, also $\Gamma$ must be a near polygon. We show that the diameter of $\Gamma$ is equal to 3 . Let $x$ be an arbitrary point of $H$. If $H \subseteq H_{x}$, then since $H$ is a maximal subspace, we must have $H=H_{x}$, contradicting the fact that every singular hyperplane of $\mathbb{E}_{3}$ admits ovoidal $W(2)$-quads. Hence, there exists a point in $\Gamma_{3}(x) \cap H$, proving the claim.

From Theorem 1.1 and Table 3, we obtain

Corollary 1.2 Let $H$ be a hyperplane of Type A2, A3, A16, B1 or B4. Then the point-line geometry induced on $\Gamma$ is a near hexagon.

Remark. As we have already mentioned above, the point-line geometry induced on a Type B1 hyperplane is a near hexagon isomorphic to $\mathbb{G}_{3}$.

\section{Dense near polygons}

In this section, we will recall some of the more advanced notions and properties of dense near polygons which we will need later.

A proper convex subspace $F$ of a dense near polygon $\Gamma$ is called big in $\Gamma$ if every point $x$ of $\Gamma$ not contained in $F$ is collinear with a unique point $\pi_{F}(x)$ of $F$. If $F$ is big in $\Gamma$ and if $H_{F}$ is a hyperplane of $F$, then the set $H:=H_{F} \cup \Gamma_{1}\left(H_{F}\right)=F \cup\left(\Gamma_{1}\left(H_{F}\right) \cap \Gamma_{1}(F)\right)$ is a hyperplane of $\Gamma$. We call $H$ the extension of $H_{F}$.

Suppose $F$ is a big convex subspace of a dense near polygon $\Gamma$ which has three points on each line. If $x \in F$, then we define $\mathcal{R}_{F}(x):=x$. If $x \notin F$, then $\mathcal{R}_{F}(x)$ denotes the unique point of the line $x \pi_{F}(x)$ different from $x$ and $\pi_{F}(x)$. The map $\mathcal{R}_{F}$ is an automorphism of $\Gamma$ and is called the reflection about $F$.

Let $\Gamma=(\mathcal{P}, \mathcal{L}, \mathrm{I})$ be a dense near polygon. A function $f: \mathcal{P} \rightarrow \mathbb{N}$ is called a valuation of $\Gamma$ if it satisfies the following properties (we call $f(x)$ the value of $x$ ): 
(V1) there exists a point with value 0 ;

(V2) every line $L$ of $\Gamma$ contains a unique point $x_{L}$ with smallest value and $f(x)=f\left(x_{L}\right)+1$ for every point $x$ of $L$ different from $x_{L}$;

(V3) every point $x$ of $\Gamma$ is contained in a (necessarily unique) convex subspace $F_{x}$ such that the following properties are satisfied for every $y \in F_{x}$ : (i) $f(y) \leq f(x)$; (ii) if $z$ is a point collinear with $y$ such that $f(z)=f(y)-1$, then $z \in F_{x}$.

Valuations of dense near polygons were introduced in De Bruyn and Vandecasteele [20]. If $f$ is a valuation of a dense near polygon $\Gamma$, then $O_{f}$ denotes the set of points of $\Gamma$ with value 0 and $G_{f}$ denotes the point-line geometry with point-set $O_{f}$, with line-set the set of all quads containing at least two points of $O_{f}$ (each such quad intersects $O_{f}$ necessarily in an ovoid), and with natural incidence. If $y$ is a point of a dense near polygon $\Gamma=(\mathcal{P}, \mathcal{L}, I)$, then the map $f: \mathcal{P} \rightarrow \mathbb{N} ; x \mapsto \mathrm{d}(x, y)$ is a valuation of $\Gamma$. We call any such valuation a classical valuation of $\Gamma$. If $f$ is a valuation of a dense near polygon $\Gamma$, then the set of points with non-maximal value is a hyperplane of $\Gamma$.

Let $\Gamma$ be a dense near hexagon and let $Q$ be a quad of $\Gamma$. If $x \in \Gamma_{1}(Q)$, then $x$ is collinear with a unique point $\pi_{Q}(x)$ of $Q$. If $x \in \Gamma_{2}(Q)$, then $O:=\Gamma_{2}(x) \cap Q$ is an ovoid of $Q$ and $\left\langle x, x_{1}\right\rangle \cap\left\langle x, x_{2}\right\rangle=\{x\}$ for any two distinct points $x_{1}$ and $x_{2}$ of $O$. We will say that the point $x$ is ovoidal with respect to $Q$. If $L$ is a line through $x$ contained in one of the quads $\left\langle x, x_{i}\right\rangle$, $x_{i} \in O$, then $L$ contains a unique point of $\Gamma_{1}(Q)$ and the remaining points of $L$ are contained in $\Gamma_{2}(Q)$. If $L$ is a line through $x$ not contained in $\bigcup_{x_{i} \in O}\left\langle x, x_{i}\right\rangle$, then $L \subseteq \Gamma_{2}(Q)$.

If $A$ is a set of points of a near polygon $\Gamma$, then we define $A^{\perp}:=\bigcap_{x \in A} x^{\perp}$ and $A^{\perp \perp}:=\left(A^{\perp}\right)^{\perp}$. If $B$ is a set of lines of a near polygon $\Gamma$, then $B^{\perp}$ denotes the set of lines of $\Gamma$ meeting each line of $B$ and $B^{\perp \perp}:=\left(B^{\perp}\right)^{\perp}$. A spread of a near polygon is a set of lines partitioning the point-set. A spread $S$ of a generalized quadrangle $Q$ is called regular if for every two distinct lines $K$ and $L$ of $S$, (i) $\{K, L\}^{\perp}$ and $\{K, L\}^{\perp \perp}$ cover the same set of points of $Q$, and (ii) $\{K, L\}^{\perp \perp} \subseteq S$.

If $x$ and $y$ are two noncollinear points of the generalized quadrangle $W(2)$, then $\{x, y\}^{\perp \perp}$ is a set of 3 points containing $x$ and $y .\{x, y\}^{\perp \perp}$ is called the hyperbolic line through $x$ and $y$. Every point of $W(2) \backslash\{x, y\}^{\perp \perp}$ is collinear with either 1 or 3 points of $\{x, y\}^{\perp \perp}$ and every ovoid of $W(2)$ intersects $\{x, y\}^{\perp \perp}$ in either 0 or 2 points.

Let $Q$ be a generalized quadrangle isomorphic to $Q(5,2)$. If $G$ is a $(3 \times 3)$-subgrid of $Q$, then there exists a unique pair $\left\{G_{1}, G_{2}\right\}$ of $(3 \times 3)$-subgrids of $Q$ such that $\left\{G, G_{1}, G_{2}\right\}$ is a partition of $Q(5,2)$ in $(3 \times 3)$-subgrids.

If $L_{1}, L_{2}, L_{3}$ and $L_{4}$ are four mutually disjoint lines of $Q(5,2)$ such that $L_{1}, L_{2}$ and $L_{3}$ are contained in a $(3 \times 3)$-grid, then $L_{1}, L_{2}, L_{3}$ and $L_{4}$ are contained in a unique regular spread of $Q(5,2)$.

We now mention some properties of the near hexagon $\mathbb{E}_{3}$. The near hexagon $\mathbb{E}_{3}$ has 567 points, 3 points on each line and 15 lines through each point. Every quad is isomorphic to either $Q(5,2)$ or $W(2)$. There are $126 Q(5,2)$-quads and $567 W(2)$-quads. Every $Q(5,2)$-quad is big. If a quad meets a $Q(5,2)$-quad $Q$, then it intersects $Q$ in either $Q$ or a line of $Q$. For every point $x$ of $\mathbb{E}_{3}$, let $\mathcal{L}_{x}$ (respectively $\mathcal{L}_{x}^{\prime}$ ) denote the point-line geometry whose points are 
the lines through $x$ and whose lines are the quads (respectively $W(2)$-quads) through $x$, with incidence being containment. Then $\mathcal{L}_{x} \cong \overline{W(2)}$ and $\mathcal{L}_{x}^{\prime} \cong W(2)$. Here, $\overline{W(2)}$ denotes the linear space obtained from $W(2)$ by adding its ovoids as extra lines.

We now define 3 additional classes of dense near hexagons with three points on each line.

(1) Let $H(5,4)$ denote a nonsingular Hermitian variety in $\operatorname{PG}(5,4)$ and let $D H(5,4)$ be the point-line geometry whose points, respectively lines, are the planes, respectively lines, contained in $H(5,4)$ (natural incidence). The point-line geometry $D H(5,4)$ is a dense near hexagon with three points on each line. It is an example of a so-called dual polar space (Cameron [6]). All quads of $D H(5,4)$ are isomorphic to $Q(5,2)$ and are big in $D H(5,4)$. By Pasini and Shpectorov [25], the near hexagon $\mathbb{E}_{3}$ can be isometrically embedded as a hyperplane in $D H(5,4)$.

(2) Again, let $H(5,4)$ denote a nonsingular Hermitian variety in $\operatorname{PG}(5,4)$. We choose a reference system in $\mathrm{PG}(5,4)$ in such a way that $X_{0}^{3}+X_{1}^{3}+\ldots+X_{5}^{3}=0$ is the equation describing $H(5,4)$. The weight of a point of $\mathrm{PG}(5,4)$ is defined as the number of its nonzero coordinates (with respect to the chosen reference system). Let $X$ denote the set of all planes of $H(5,4)$ which contain a point with weight 2 . Then $X$ is a subspace of the dual polar space $D H(5,4)$ associated with $H(5,4)$. By Brouwer et al. [3], the point-line geometry induced on that subspace is a dense near hexagon with three points on each line and 12 lines through each point. We will denote this near hexagon by $\mathbb{G}_{3}$. The above description yields an isometric embedding of $\mathbb{G}_{3}$ into $D H(5,4)$. Every quad of $\mathbb{G}_{3}$ is isomorphic to either the $(3 \times 3)$-grid, $W(2)$ or $Q(5,2)$. $\mathbb{G}_{3}$ has 405 points, 405 grid-quads, $243 W(2)$-quads and $45 Q(5,2)$-quads. Every $Q(5,2)$-quad is big. The automorphism group of $\mathbb{G}_{3}$ has two orbits on the set of lines. A line of $\mathbb{G}_{3}$ is called special if it is contained in $2 Q(5,2)$-quads, $0 W(2)$-quads and 3 grid-quads. A line of $\mathbb{G}_{3}$ is called ordinary if it is contained in $1 Q(5,2)$-quad, $3 W(2)$-quads and 1 grid-quad. Every point of $\mathbb{G}_{3}$ is contained in 3 special lines and 9 ordinary lines.

(3) The near hexagon $Q(5,2) \otimes Q(5,2)$ is an example of a so-called glued near hexagon ([9]). It is a dense near hexagon with three points per line and 9 lines through each point. There are 243 vertices and every quad is isomorphic to either the $(3 \times 3)$-grid or $Q(5,2)$. Every $Q(5,2)$-quad is big. The set of $18 Q(5,2)$-quads can be partitioned into two families $\mathcal{F}_{1}$ and $\mathcal{F}_{2}$ such that

(i) every quad of $\mathcal{F}_{1}$ intersects every quad of $\mathcal{F}_{2}$ in a line;

(ii) the set $S^{*}=\left\{Q_{1} \cap Q_{2} \mid Q_{1} \in \mathcal{F}_{1}, Q_{2} \in \mathcal{F}_{2}\right\}$ is a spread of $Q(5,2) \otimes Q(5,2)$;

(iii) every line of $Q(5,2) \otimes Q(5,2)$ is contained in a quad of $\mathcal{F}_{1} \cup \mathcal{F}_{2}$;

(iv) for a given $Q \in \mathcal{F}_{i}, i \in\{1,2\}$, the set $S_{Q}:=\left\{Q \cap Q_{3-i} \mid Q_{3-i} \in \mathcal{F}_{3-i}\right\}$ is a regular spread of $Q$.

Conversely, every dense near hexagon with three points per line admitting two partitions $\mathcal{F}_{1}$ and $\mathcal{F}_{2}$ in $Q(5,2)$-quads satisfying (i), (ii), (iii), (iv) above is isomorphic to $Q(5,2) \otimes Q(5,2)$.

By Cooperstein [8] and De Bruyn [12], there exists a nice full polarized embedding $e^{\prime}$ of the dual polar space $D H(5,4)$ into the projective space $\mathrm{PG}(19,2)$. We refer to this particular 
embedding as the Grassmann-embedding of $D H(5,4)$. Now, suppose $\mathbb{E}_{3}$ is (isometrically) embedded as a hyperplane in $D H(5,4)$. De Bruyn and Pralle [17], [16] showed that $\mathbb{E}_{3}$ cannot arise from $e^{\prime}$. Hence, $\left\langle e^{\prime}\left(\mathbb{E}_{3}\right)\right\rangle=\mathrm{PG}(19,2)$ and $e^{\prime}$ induces a full polarized embedding $\bar{e}$ of $\mathbb{E}_{3}$ into PG $(19,2)$. For every point $x$ of $\mathbb{E}_{3}$, let $H_{x}$ (respectively $H_{x}^{\prime}$ ) denote the singular hyperplane of $\mathbb{E}_{3}$ (respectively $D H(5,4)$ ) with deepest point $x$. Then $H_{x}=H_{x}^{\prime} \cap \mathbb{E}_{3}$.

Proposition 2.1 The embedding $\bar{e}$ is the minimal full polarized embedding of $\mathbb{E}_{3}$.

Proof. Let $x_{1}, x_{2}, \ldots, x_{20}$ be 20 points of $\mathbb{E}_{3}$ such that $\left\langle e^{\prime}\left(x_{1}\right), \ldots, e^{\prime}\left(x_{20}\right)\right\rangle=\operatorname{PG}(19,2)$. By Cardinali, De Bruyn and Pasini [7, Section 4.2], $k \geq 2$ points $e^{\prime}\left(y_{1}\right), e^{\prime}\left(y_{2}\right), \ldots, e^{\prime}\left(y_{k}\right)$ of $\mathrm{PG}(19,2)$ are linearly independent if and only if the hyperplanes $\left\langle e^{\prime}\left(H_{y_{1}}^{\prime}\right)\right\rangle,\left\langle e^{\prime}\left(H_{y_{2}}^{\prime}\right)\right\rangle, \ldots$, $\left\langle e^{\prime}\left(H_{y_{k}}^{\prime}\right)\right\rangle$ of $\mathrm{PG}(19,2)$ are linearly independent. It follows that $\bigcap_{1 \leq i \leq 20}\left\langle e^{\prime}\left(H_{x_{i}}^{\prime}\right)\right\rangle=\emptyset$. Now, for every $i \in\{1, \ldots, 20\},\left\langle\bar{e}\left(H_{x_{i}}\right)\right\rangle=\left\langle e^{\prime}\left(H_{x_{i}}^{\prime}\right)\right\rangle$. Hence, $\bigcap_{x \in \mathbb{E}_{3}}\left\langle\bar{e}\left(H_{x}\right)\right\rangle=\emptyset$, implying that $\bar{e}$ is the minimal full polarized embedding of $\mathbb{E}_{3}$.

\section{$3 \quad$ The basic hyperplane classes of $\mathbb{E}_{3}$}

The aim of this section is to define five classes of hyperplanes of $\mathbb{E}_{3}$. These classes are called the basic hyperplane classes of $\mathbb{E}_{3}$. We will construct all the remaining hyperplane classes from them. It is straightforward to calculate some combinatorial properties of a representative of each of these five classes (like $N, v, D E$ ). In this way we were able to identify the five hyperplane classes with five orbits found in our computer search.

Definition. A hyperplane of $\mathbb{E}_{3}$ is said to be of Type $A 1$ if it is a singular hyperplane of $\mathbb{E}_{3}$. As said before, if $x$ is a point of $\mathbb{E}_{3}$, then we denote by $H_{x}$ the singular hyperplane of $\mathbb{E}_{3}$ with deepest point $x$.

Proposition 3.1 If $W$ is a $W(2)$-quad of $\mathbb{E}_{3}$, then $W \cup \Gamma_{1}(W)$ is a hyperplane of $\mathbb{E}_{3}$.

Proof. Let $x$ be an arbitrary point of $\Gamma_{2}(W)$ and let $L$ be an arbitrary line through $x$. The 5 $W(2)$-quads through $x$ which meet $W$ in a point of the ovoid $\Gamma_{2}(x) \cap W$ of $W$ partition the set of 15 lines through $x$. Hence $L$, which is contained in precisely one of these quads, contains a unique point of $\Gamma_{1}(W)$. This proves that $W \cup \Gamma_{1}(W)$ is a hyperplane of $\mathbb{E}_{3}$.

Definitions. - A hyperplane $H$ of $\mathbb{E}_{3}$ is said to be of Type $A 2$ if $H=W \cup \Gamma_{1}(W)$ for some $W(2)$-quad $W$ of $\mathbb{E}_{3}$. We will denote such a hyperplane by $H_{W}$.

- A hyperplane of $\mathbb{E}_{3}$ is said to be of Type $A 3$ if it is the extension of a $W(2)$-subquadrangle $W$ of a $Q(5,2)$-quad $Q$. We will also denote such a hyperplane by $H_{W}$. Notice that also in this case $H_{W}=W \cup \Gamma_{1}(W)$.

By De Bruyn and Vandecasteele [21, Section 7.3], the near hexagon $\mathbb{E}_{3}$ has up to isomorphism a unique non-classical valuation. Any such valuation can be obtained as follows. Let $W$ 
be a $W(2)$-quad of $\mathbb{E}_{3}$ and let $z \in \Gamma_{2}(W)$. Put $\Gamma_{2}(z) \cap W=\left\{z_{1}, z_{2}, z_{3}, z_{4}, z_{5}\right\}$ and let $O_{i}$, $i \in\{1,2,3,4,5\}$, denote the unique ovoid of the $W(2)$-quad $\left\langle z, z_{i}\right\rangle$ containing the points $z$ and $z_{i}$. Put $Z:=O_{1} \cup O_{2} \cup \cdots \cup O_{5}$ and $f(x):=\mathrm{d}(x, Z)$ for every point $x$ of $\mathbb{E}_{3}$. Then $f$ is a non-classical valuation of $\mathbb{E}_{3}$ with $O_{f}=Z$ and $G_{f} \cong \mathrm{PG}(2,4)$. A non-classical valuation of $\mathbb{E}_{3}$ has 21 points with value 0,210 points with value 1 and 336 points with value 2 . The points with value 0 and 1 define a hyperplane $H_{f}$ of $\mathbb{E}_{3}$.

Definition. A hyperplane $H$ of $\mathbb{E}_{3}$ is said to be of Type $A 4$ if $H=H_{f}$ for some non-classical valuation $f$ of $\mathbb{E}_{3}$.

The hyperplane $H_{f}$ can also be obtained as follows, see [21, Section 7.3]. Embed $\mathbb{E}_{3}$ isometrically into the dual polar space $D H(5,4)$. Then there exists a unique point $x$ in $D H(5,4) \backslash \mathbb{E}_{3}$ such that $x^{\perp} \cap \mathbb{E}_{3}=O_{f}$ and $H_{f}=H_{x}^{\prime} \cap \mathbb{E}_{3}$, where $H_{x}^{\prime}$ denotes the singular hyperplane of $D H(5,4)$ with deepest point $x$.

Proposition 3.2 The near hexagon $\mathbb{G}_{3}$ can be embedded as a hyperplane into $\mathbb{E}_{3}$.

Proof. By Brouwer et al. [3], the near hexagon $\mathbb{G}_{3}$ can be isometrically embedded into the dual polar space $D H(5,4)$. The absolutely universal embedding of $D H(5,4)$ has vector dimension 22, see e.g. Yoshiara [31] or Li [24]. If $\widetilde{e}^{\prime}$ denotes the absolutely universal embedding of $D H(5,4)$ into $\mathrm{PG}(21,2)$, then $\widetilde{e}^{\prime}$ induces an embedding $e$ of $\mathbb{G}_{3}$ into the subspace $\left\langle\widetilde{e}^{\prime}\left(\mathbb{G}_{3}\right)\right\rangle$ of $\mathrm{PG}(21,2)$. Since $\widetilde{e}$ is polarized and the embedding of $\mathbb{G}_{3}$ into $D H(5,4)$ is isometric, also $e$ has to be polarized. In De Bruyn [11], it has been shown that there exists a unique full polarized embedding of $\mathbb{G}_{3}$. This embedding has vector dimension 20 . So, $\operatorname{dim}\left\langle\widetilde{e}^{\prime}\left(\mathbb{G}_{3}\right)\right\rangle=19$. Now, let $\Pi$ be one of the three hyperplanes of $\operatorname{PG}(21,2)$ through $\left\langle\widetilde{e}^{\prime}\left(\mathbb{G}_{3}\right)\right\rangle$ and let $H$ be the hyperplane of $D H(5,4)$ arising from $\Pi$. Now, for every quad $Q$ of $\mathbb{G}_{3}$, there exists a unique quad $\bar{Q}$ in $D H(5,4)$ such that $\bar{Q} \cap \mathbb{G}_{3}=Q(\bar{Q}$ is the smallest convex subspace of $D H(5,4)$ containing $Q)$. Since both $\mathbb{G}_{3}$ and $D H(5,4)$ have precisely 693 quads, every quad of $D H(5,4)$ intersects $\mathbb{G}_{3}$ in a quad. Now, if $Q$ is a quad of $D H(5,4)$, then $Q \cap H$ contains the quad $Q \cap \mathbb{G}_{3}$ of $\mathbb{G}_{3}$. It follows that $H$ is a locally subquadrangular hyperplane of $D H(5,4)$. By Pasini and Shpectorov [25], there exists up to isomorphism a unique locally subquadrangular hyperplane in $D H(5,4)$. The point-line geometry induced on such a locally subquadrangular hyperplane is a near hexagon isomorphic to $\mathbb{E}_{3}$. Since $\left\langle\tilde{e}^{\prime}\left(\mathbb{G}_{3}\right)\right\rangle$ is a hyperplane of $\Pi$, every line of $H$ meets $\mathbb{G}_{3}$, proving the lemma.

Definition. Every hyperplane of $\mathbb{E}_{3}$ carrying the structure of a $\mathbb{G}_{3}$ near hexagon is called a hyperplane of Type B1.

A direct construction of the hyperplanes of Type B1 in the near hexagon $\mathbb{E}_{3}$ can be given by relying on De Bruyn [10, Section 3]. Let $Q_{1}$ and $Q_{2}$ be two disjoint $Q(5,2)$-quads of $\mathbb{E}_{3}$, put $Q_{3}:=\mathcal{R}_{Q_{1}}\left(Q_{2}\right)=\mathcal{R}_{Q_{2}}\left(Q_{1}\right)$ and let $\mathcal{V}$ denote the set of all $Q(5,2)$-quads meeting $Q_{1}$ and $Q_{2}$. Then $|\mathcal{V}|=18$. Moreover, the quad $Q_{1}$ can be partitioned into 3 subgrids $G_{1}, G_{2}$ and $G_{3}$ such 
that every line $Q_{1} \cap Q$ with $Q \in \mathcal{V}$ is contained in either $G_{1}, G_{2}$ or $G_{3}$. In [10, Lemma 5] it was shown that $Q_{1} \cup Q_{2} \cup Q_{3} \cup \cup_{Q \in \mathcal{V}} Q$ is a hyperplane of $\mathbb{E}_{3}$. The hyperplanes of $\mathbb{E}_{3}$ which can be obtained in this way are precisely the Type B1 hyperplanes.

\section{The mutual position of two $W(2)$-quads}

In this section, we study the mutual position of two distinct $W(2)$-quads $W_{1}$ and $W_{2}$. In almost all possible cases, we give an alternative description of the hyperplane $H_{W_{1}} * H_{W_{2}}$. We will need some of these alternative descriptions in Section 5.

Proposition 4.1 One of the following 5 cases occurs for two $W(2)$-quads $W_{1}$ and $W_{2}$ of $\mathbb{E}_{3}$ :

(1) $W_{1}=W_{2}$;

(2) $W_{1} \cap W_{2}$ is a line;

(3) $W_{1} \cap W_{2}$ is a point;

(4) $W_{2} \subseteq \Gamma_{1}\left(W_{1}\right)$ and there exists a unique $Q(5,2)$-quad $Q$ such that $W_{2}=\mathcal{R}_{Q}\left(W_{1}\right)$;

(5) $W_{1} \cap W_{2}=\emptyset, W_{2} \cap \Gamma_{1}\left(W_{1}\right)$ is a $(3 \times 3)$-subgrid of $W_{2}$ and $W_{1} \cap \Gamma_{1}\left(W_{2}\right)$ is a $(3 \times 3)$-subgrid of $W_{1}$.

Proof. If $W_{1} \cap W_{2} \neq \emptyset$, then obviously one of the cases (1), (2), (3) occurs. So, suppose $W_{1} \cap W_{2}=\emptyset$. Since $W_{1} \cup \Gamma_{1}\left(W_{1}\right)$ is a hyperplane of $\mathbb{E}_{3}, \Gamma_{1}\left(W_{1}\right) \cap W_{2}$ is either $W_{2}$ or a hyperplane of $W_{2}$.

Suppose the former case occurs. Then we show that there exists at most $1 Q(5,2)$-quad $Q$ such that $W_{2}=\mathcal{R}_{Q}\left(W_{1}\right)$. For, consider all 15 lines which meet $W_{1}$ and $W_{2}$ and let $W_{3}$ denote the set of all 15 points outside $W_{1} \cup W_{2}$ which are contained in one of these lines. Then $W_{3}$ is a subspace which carries the structure of a generalized quadrangle isomorphic to $W(2)$. So, either $W_{3}$ is a $W(2)$-quad or is properly contained in a $Q(5,2)$-quad which then necessarily coincides with $\left\langle W_{3}\right\rangle$. It follows that if $Q$ is a $Q(5,2)$-quad such that $W_{2}=\mathcal{R}_{Q}\left(W_{1}\right)$, then $Q=\left\langle W_{3}\right\rangle$.

Suppose the latter case occurs. Let $x$ be an arbitrary point of $W_{2} \cap \Gamma_{1}\left(W_{1}\right)$ and let $x^{\prime}$ denote the unique point of $W_{1}$ collinear with $x$. There are two $Q(5,2)$-quads through the line $x x^{\prime}$. If $Q$ is such a $Q(5,2)$-quad, then $Q \cap W_{1}$ and $Q \cap W_{2}$ are lines. Moreover, every point of $Q \cap W_{2}$ has distance 1 from a unique point of $Q \cap W_{1}$. It follows that every point of $\Gamma_{1}\left(W_{1}\right) \cap W_{2}$ is contained in at least 2 lines which are contained in $\Gamma_{1}\left(W_{1}\right) \cap W_{2}$. This is only possible when the hyperplane $\Gamma_{1}\left(W_{1}\right) \cap W_{2}$ of $W_{2}$ is a $(3 \times 3)$-subgrid.

Now, fix $W_{1}$ and let $W_{2}$ range over all $567 W(2)$-quads of $\mathbb{E}_{3}$. Let $N_{i}, i \in\{1,2,3,4,5\}$, denote the number of times case (i) of the lemma occurs. We calculate the $N_{i}$ 's. Obviously, $N_{1}=1$. Through each of the 15 lines of $W_{1}$, there are $2 W(2)$-quads which intersect $W_{1}$ in a line. Hence, $N_{2}=15 \cdot 2=30$. Through each of the 15 points of $W_{1}$, there are $8 W(2)$-quads which intersect $W_{1}$ in a unique point. Hence, $N_{3}=15 \cdot 8=120$. The $W(2)$-quads $W_{2}$ of $\mathbb{E}_{3}$ such that $\left(W_{1}, W_{2}\right)$ satisfies property (4) of the lemma are in bijective correspondence with the $Q(5,2)$-quads disjoint from $W_{1}$. Since there are 96 such $Q(5,2)$-quads, we have $N_{4}=96$. In order to calculate $N_{5}$, we count in two different ways the pairs $\left(x, W_{2}\right)$ where $x \in \Gamma_{2}\left(W_{1}\right)$ 
and $W_{2}$ is a $W(2)$-quad through $x$ disjoint from $W_{1}$. There are $\left|\Gamma_{2}\left(W_{1}\right)\right|=192$ possibilities for $x$, and for given $x$, there are $10\left(=15-\left|\Gamma_{2}(x) \cap W_{1}\right|\right)$ possibilities for $W_{2}$. On the other hand, there are $N_{5}$ possibilities for $W_{2}$ and for given $W_{2}$ there are 6 possibilities for $x$. Hence, $N_{5}=\frac{192 \cdot 10}{6}=320$.

The lemma now follows from the fact that $N_{1}+N_{2}+N_{3}+N_{4}+N_{5}=1+30+120+96+320=$ 567 equals the total number of $W(2)$-quads of $\mathbb{E}_{3}$.

Proposition 4.2 Let $W_{1}, W_{2}$ and $W_{3}$ denote the three $W(2)$-quads of $\mathbb{E}_{3}$ through a given line $L$ of $\mathbb{E}_{3}$. Then $H_{W_{1}} * H_{W_{2}}=H_{W_{3}}$.

Proof. Since hyperplanes are maximal subspaces it suffices to show that $H_{W_{3}} \subseteq H_{W_{1}} * H_{W_{2}}$. Since $W_{3} \subseteq H_{W_{1}} \cap H_{W_{2}}, W_{3} \subseteq H_{W_{1}} * H_{W_{2}}$. Now, let $x$ denote an arbitrary point of $\Gamma_{1}\left(W_{3}\right)$ and let $x^{\prime}$ denote the unique point of $W_{3}$ collinear with $x$. If $x^{\prime} \in L$, then $x \in H_{W_{1}} \cap H_{W_{2}} \subseteq H_{W_{1}} * H_{W_{2}}$. So, suppose $x^{\prime} \notin L$. Let $x^{\prime \prime}$ denote the unique point of $L$ collinear with $x^{\prime}$. We distinguish two cases:

(1) Suppose $Q:=\left\langle x^{\prime \prime} x^{\prime}, x^{\prime} x\right\rangle$ is a $Q(5,2)$-quad. Then $Q \cap W_{1}$ and $Q \cap W_{2}$ are lines. Every point of $x x^{\prime}$ has distance 1 from a unique point of $Q \cap W_{1}$ and a unique point of $Q \cap W_{2}$. Hence, $x \in H_{W_{1}} \cap H_{W_{2}} \subseteq H_{W_{1}} * H_{W_{2}}$.

(2) Suppose $Q:=\left\langle x^{\prime \prime} x^{\prime}, x^{\prime} x\right\rangle$ is a $W(2)$-quad. Since $\mathcal{L}_{x^{\prime \prime}}^{\prime} \cong W(2), Q$ intersects both $W_{1}$ and $W_{2}$ in only the point $x^{\prime \prime}$. It follows that $x \notin H_{W_{1}}$ and $x \notin H_{W_{2}}$. Hence, $x \in H_{W_{1}} * H_{W_{2}}$.

Proposition 4.3 Let $W_{1}$ and $W_{2}$ denote two $W(2)$-quads of $\mathbb{E}_{3}$ intersecting in a unique point $x$. Let $W_{3}$ denote the unique $W(2)$-quad of $\mathbb{E}_{3}$ through $x$ such that $(i) W_{3} \cap W_{1}=W_{3} \cap W_{2}=\{x\}$; (ii) every $W(2)$-quad intersecting each of $W_{1}, W_{2}$ in a line also intersects $W_{3}$ in a line. $\left(W_{3}\right.$ is the unique element of the set $\left\{W_{1}, W_{2}\right\}^{\perp \perp} \backslash\left\{W_{1}, W_{2}\right\}$, where $W_{1}$ and $W_{2}$ are regarded as lines of the generalized quadrangle $\mathcal{L}_{x}^{\prime} \cong W(2)$.) Then $H_{W_{1}} * H_{W_{2}}=H_{W_{3}} * H_{x}$.

Proof. We show that $H_{W_{1}} * H_{W_{2}} * H_{W_{3}} * H_{x}$ coincides with the whole point-set of $\mathbb{E}_{3}$. Let $y$ be a point of $\mathbb{E}_{3}$.

If $\mathrm{d}(x, y) \leq 1$, then $y \in H_{W_{1}} \cap H_{W_{2}} \cap H_{W_{3}} \cap H_{x}$ and hence $y \in H_{W_{1}} * H_{W_{2}} * H_{W_{3}} * H_{x}$.

Suppose $\mathrm{d}(x, y)=2$. We distinguish four cases:

(1) $y$ is contained in one of $W_{1}, W_{2}, W_{3}$. Without loss of generality, we may suppose that $y$ is contained in $W_{1}$. Then $y \in H_{W_{1}} \cap H_{x}$ and $y \notin H_{W_{2}} \cup H_{W_{3}}$. This implies that $y \in$ $H_{W_{1}} * H_{W_{2}} * H_{W_{3}} * H_{x}$.

(2) $\langle x, y\rangle$ is a $W(2)$-quad which intersects each of $W_{1}, W_{2}, W_{3}$ in a line. Then $y \in H_{W_{1}} \cap$ $H_{W_{2}} \cap H_{W_{3}} \cap H_{x} \subseteq H_{W_{1}} * H_{W_{2}} * H_{W_{3}} * H_{x}$.

(3) $\langle x, y\rangle$ is a $W(2)$-quad which intersects precisely 1 of $W_{1}, W_{2}, W_{3}$ in a line and the two others in the point $x$. Without loss of generality, we may suppose that $\langle x, y\rangle \cap W_{1}$ is a line and $\langle x, y\rangle \cap W_{2}=\langle x, y\rangle \cap W_{3}=\{x\}$. Then $y \in H_{W_{1}} \cap H_{x}$ and $y \notin H_{W_{2}} \cup H_{W_{3}}$. It follows that $y \in H_{W_{1}} * H_{W_{2}} * H_{W_{3}} * H_{x}$.

(4) $\langle x, y\rangle$ is a $Q(5,2)$-quad. Then $\langle x, y\rangle \cap W_{i}$ is a line for every $i \in\{1,2,3\}$. Hence, $y \in H_{W_{1}} \cap H_{W_{2}} \cap H_{W_{3}} \cap H_{x} \subseteq H_{W_{1}} * H_{W_{2}} * H_{W_{3}} * H_{x}$. 
From the above discussion it follows that the singular hyperplane $H_{x}$ with deepest point $x$ is contained in $H_{W_{1}} * H_{W_{2}} * H_{W_{3}} * H_{x}$. Since $H_{x}$ is a maximal subspace of $\mathbb{E}_{3}$, it suffices to show that there exists a point $z$ at distance 3 from $x$ which is contained in $H_{W_{1}} * H_{W_{2}} * H_{W_{3}} * H_{x}$.

Let $y_{1}$ be an arbitrary point of $W_{1}$ at distance 2 from $x$. Then $y_{1}$ is ovoidal with respect to $W_{2}$. Let $y_{2}$ denote an arbitrary point of $\Gamma_{2}\left(y_{1}\right) \cap W_{2} \backslash\{x\}$. Let $W$ be the quad $\left\langle y_{1}, y_{2}\right\rangle$. The point $x$ is ovoidal with respect to $W$. Put $\Gamma_{2}(x) \cap W=\left\{y_{1}, y_{2}, y_{3}, y_{4}, y_{5}\right\}$. For every $i \in\{3,4,5\}$, put $R_{i}:=\left\langle x, y_{i}\right\rangle$. The quad $R_{i}$ intersects $W_{3}$ in a line. Hence, $y_{3}, y_{4}, y_{5} \in \Gamma_{1}\left(W_{3}\right)$. Now, since $y_{1} \notin \Gamma_{1}\left(W_{3}\right), \Gamma_{1}\left(W_{3}\right) \cap W$ is a $(3 \times 3)$-subgrid $G$ of $W$ and $\Gamma_{1}(W) \cap W_{3}$ is a $(3 \times 3)$-subgrid $G^{\prime}$ of $W_{3}$. Clearly, $\left\{y_{3}, y_{4}, y_{5}\right\}$ is an ovoid of $G$. Now, let $z$ be an arbitrary point of $W$ collinear with $y_{1}$ and $y_{2}$. Then $z \in H_{W_{1}} \cap H_{W_{2}}$ and $z \notin H_{x}$. Since $\left\{y_{1}, y_{2}, y_{3}, y_{4}, y_{5}\right\}$ is an ovoid of $W$, $z$ is collinear with precisely one point of $\left\{y_{3}, y_{4}, y_{5}\right\}$. Hence $z \notin G$, i.e. $z \notin \Gamma_{1}\left(W_{3}\right)$. Since $z \in H_{W_{1}} \cap H_{W_{2}}$ and $z \notin H_{W_{3}} \cup H_{x}$, the point $z$ belongs to $H_{W_{1}} * H_{W_{2}} * H_{W_{3}} * H_{z}$. As said before, this implies that $H_{W_{1}} * H_{W_{2}} * H_{W_{3}} * H_{z}$ coincides with the whole point-set of $\mathbb{E}_{3}$. So, we have that $H_{W_{1}} * H_{W_{2}}=H_{W_{3}} * H_{x}$.

Proposition 4.4 Let $Q$ be a $Q(5,2)$-quad of $\mathbb{E}_{3}$ and let $W_{1}$ be a $W(2)$-quad disjoint from $Q$. Put $W_{2}:=\mathcal{R}_{Q}\left(W_{1}\right)$ and $W_{3}:=\pi_{Q}\left(W_{1}\right)=\pi_{Q}\left(W_{2}\right)$. Then $H_{W_{1}} * H_{W_{2}}=H_{W_{3}}$.

Proof. Since $W_{3} \subseteq H_{W_{1}} \cap H_{W_{2}}, W_{3} \subseteq H_{W_{1}} * H_{W_{2}}$.

Let $x$ be an arbitrary point of $Q \backslash W_{3}$. Since $x \notin H_{W_{1}} \cup H_{W_{2}}$, we have $x \in H_{W_{1}} * H_{W_{2}}$.

Let $L$ be an arbitrary line of $\mathbb{E}_{3}$ which intersects $Q$ in a point belonging to $W_{3}$. If $L$ meets $W_{1}$ and $W_{2}$, then $L \subseteq H_{W_{1}} \cap H_{W_{2}}$ and hence $L \subseteq H_{W_{1}} * H_{W_{2}}$. Suppose therefore that $L$ does not meet $W_{1} \cup W_{2}$. Then $L \neq L^{\prime}$, where $L^{\prime}$ is the unique line through $L \cap Q$ meeting $W_{1}$ and $W_{2}$. The quad $\left\langle L, L^{\prime}\right\rangle$ intersects $Q$ in a line. If this line is contained in $W_{3}$, then $L \subseteq H_{W_{1}} \cap H_{W_{2}}$ and hence $L \subseteq H_{W_{1}} * H_{W_{2}}$. If this line is not contained in $W_{3}$, then no point of $L \backslash Q$ belongs to $H_{W_{1}} \cup H_{W_{2}}$ and hence also in this case we have $L \subseteq H_{W_{1}} * H_{W_{2}}$.

From the above we know that $H_{W_{3}} \subseteq H_{W_{1}} * H_{W_{2}}$. Now, $H_{W_{3}}$ is a maximal subspace of $\mathbb{E}_{3}$ and $H_{W_{1}} * H_{W_{2}}$ is a proper subspace of $\mathbb{E}_{3}$ since $H_{W_{1}} \neq H_{W_{2}}$. Hence, $H_{W_{3}}=H_{W_{1}} * H_{W_{2}}$.

\section{Explicit constructions of a representative of the re- maining hyperplane classes}

\subsection{Two lemmas}

We leave the proofs of the following two lemmas as straightforward exercises to the reader.

Lemma 5.1 Let $G_{1}$ and $G_{2}$ be two distinct hyperplanes of $Q(5,2)$.

(i) If $G_{1}$ and $G_{2}$ are two singular hyperplanes whose respective deepest points $x_{1}$ and $x_{2}$ lie on a line $L$, then $G_{1} * G_{2}$ is the singular hyperplane whose deepest point is the unique point in $L \backslash\left\{x_{1}, x_{2}\right\}$. 
(ii) If $G_{1}$ and $G_{2}$ are two singular hyperplanes whose deepest points lie at distance 2 from each other, then $G_{1} * G_{2}$ is a $W(2)$-subquadrangle of $Q(5,2)$.

(iii) If $G_{1}$ is a $W(2)$-subquadrangle of $Q(5,2)$ and if $G_{2}$ is a singular hyperplane of $Q(5,2)$ whose deepest point lies in $G_{1}$, then $G_{1} * G_{2}$ is a $W(2)$-subquadrangle of $Q(5,2)$.

(iv) If $G_{1}$ is a $W(2)$-subquadrangle of $Q(5,2)$ and if $G_{2}$ is a singular hyperplane of $Q(5,2)$ whose deepest point $x_{2}$ lies outside $G_{1}$, then $G_{1} * G_{2}$ is a singular hyperplane of $Q(5,2)$ whose deepest point lies in $\Gamma_{2}\left(x_{2}\right) \backslash G_{1}$.

(v) If $G_{1}$ and $G_{2}$ are two $W(2)$-subquadrangles of $Q(5,2)$ intersecting in a $(3 \times 3)$-subgrid of both $G_{1}$ and $G_{2}$, then $G_{1} * G_{2}$ is a $W(2)$-subquadrangle of $Q(5,2)$.

(vi) If $G_{1}$ and $G_{2}$ are two $W(2)$-subquadrangles intersecting in the union of three lines through a point $x$, then $G_{1} * G_{2}$ is the singular hyperplane of $Q(5,2)$ with deepest point $x$.

Lemma 5.2 Let $Q$ be a $Q(5,2)$-quad of $\mathbb{E}_{3}$ and let $G_{1}$ and $G_{2}$ be two distinct hyperplanes of $Q$. Let $H_{i}, i \in\{1,2\}$, be the hyperplane of $\mathbb{E}_{3}$ obtained by extending $G_{i}$. Then $H_{1} * H_{2}$ coincides with the extension of the hyperplane $G_{1} * G_{2}$ of $Q$.

\subsection{Hyperplanes of the form $H_{x_{1}} * H_{x_{2}}$}

In this subsection, we give an interpretation to the entries occurring in line "A1" of Table 1. This allows us to identify two extra classes of hyperplanes (A5 and A6).

Proposition 5.3 Let $x_{1}$ and $x_{2}$ be two points of $\mathbb{E}_{3}$.

(i) If $d\left(x_{1}, x_{2}\right)=1$, then $H_{x_{1}} * H_{x_{2}}=H_{x_{3}}$, where $x_{3}$ is the third point of the line $x_{1} x_{2}$.

(ii) If $d\left(x_{1}, x_{2}\right)=2$ and $\left\langle x_{1}, x_{2}\right\rangle$ is a $Q(5,2)$-quad, then $H_{x_{1}} * H_{x_{2}}$ is the extension of a $W(2)$-subquadrangle of $\left\langle x_{1}, x_{2}\right\rangle$.

Proof. This follows from Lemmas 5.1 and 5.2. (For case (i), take an arbitrary Q(5,2)-quad through the line $x_{1} x_{2}$.)

For a given point $x_{1}$, there are 30 points at distance 1 from $x_{1}, 120$ points $x_{2} \in \Gamma_{2}\left(x_{1}\right)$ for which $\left\langle x_{1}, x_{2}\right\rangle$ is a $W(2)$-quad, 96 points $x_{2}^{\prime} \in \Gamma_{2}\left(x_{1}\right)$ for which $\left\langle x_{1}, x_{2}^{\prime}\right\rangle$ is a $Q(5,2)$-quad and 320 points at distance 3 from $x_{1}$. If $x_{2} \in \Gamma_{2}\left(x_{1}\right)$ such that $\left\langle x_{1}, x_{2}\right\rangle$ is a $W(2)$-quad and $x \in \Gamma_{1}\left(x_{1}\right) \cap \Gamma_{1}\left(x_{2}\right)$, then each line through $x$ is contained in $H_{x_{1}} * H_{x_{2}}$. Line "A1" of Table 1, the line distribution mentioned in Table 3 and Proposition 5.3 now allow us to identify the two extra hyperplane classes.

Definitions. - A hyperplane of $\mathbb{E}_{3}$ is said to be of Type $A 5$ if it is of the form $H_{x_{1}} * H_{x_{2}}$, where $x_{1}$ and $x_{2}$ are two points of $\mathbb{E}_{3}$ satisfying $\mathrm{d}\left(x_{1}, x_{2}\right)=2$ and $\left\langle x_{1}, x_{2}\right\rangle$ is a $W(2)$-quad.

- A hyperplane of $\mathbb{E}_{3}$ is said to be of Type $A 6$ if it is of the form $H_{x_{1}} * H_{x_{2}}$ where $x_{1}$ and $x_{2}$ are two points of $\mathbb{E}_{3}$ at distance 3 from each other. 
Two other easy constructions for a Type A5 hyperplane will be given in Corollary 5.5. We will meet other constructions for a Type A6 hyperplane. The description mentioned in Proposition 5.6(iii) can compete in simplicity with the one given above.

\subsection{Hyperplanes of the form $H_{x} * H_{W}, W$ a $W(2)$-quad}

In this subsection, we give an interpretation to the entries occurring in line "A2" of Table 1. This allows us to identify two extra classes of hyperplanes (A7 and A8).

Proposition 5.4 If $\left\{x_{1}, x_{2}, x_{3}\right\}$ is a hyperbolic line of a $W(2)$-quad $W$, then $H_{x_{1}} * H_{x_{2}} * H_{x_{3}}=$ $H_{W}$.

Proof. Since $W \subseteq H_{x_{1}} \cap H_{x_{2}} \cap H_{x_{3}}, W \subseteq H_{x_{1}} * H_{x_{2}} * H_{x_{3}}$.

Let $x$ be an arbitrary point of $\Gamma_{1}(W)$ and let $x^{\prime}$ denote the unique point of $W$ collinear with $x$. If $x^{\prime} \in\left\{x_{1}, x_{2}, x_{3}\right\}$, then $x$ has distance 1 from $x^{\prime}$ and distance 3 from each point of $\left\{x_{1}, x_{2}, x_{3}\right\} \backslash\left\{x^{\prime}\right\}$, implying that $x \in H_{x_{1}} * H_{x_{2}} * H_{x_{3}}$. If $x^{\prime} \notin\left\{x_{1}, x_{2}, x_{3}\right\}$, then $x^{\prime}$ has distance 1 from either 1 or 3 points of $\left\{x_{1}, x_{2}, x_{3}\right\}$. Hence, $x$ has distance 2 from either 1 or 3 points of $\left\{x_{1}, x_{2}, x_{3}\right\}$ and distance 3 from the other points of $\left\{x_{1}, x_{2}, x_{3}\right\}$. This again implies that $x \in H_{x_{1}} * H_{x_{2}} * H_{x_{3}}$.

Let $x$ be an arbitrary point of $\Gamma_{2}(W)$. Then the ovoid $\Gamma_{2}(x) \cap W$ of $W$ and the hyperbolic line $\left\{x_{1}, x_{2}, x_{3}\right\}$ of $W$ intersect in either 0 or 2 points. In either case, we have $x \notin H_{x_{1}} * H_{x_{2}} * H_{x_{3}}$.

Corollary 5.5 ( $i$ ) If $W$ is a $W(2)$-quad of $\mathbb{E}_{3}$ and $x \in W$, then $H_{x} * H_{W}$ is a hyperplane of Type A5.

(ii) If $W_{1}$ and $W_{2}$ are two $W(2)$-quads meeting in a singleton $\{x\}$, then $H_{W_{1}} * H_{W_{2}}$ is a hyperplane of Type A5.

Proof. Part (i) follows from the fact that the equality $H_{x_{1}} * H_{x_{2}} * H_{x_{3}}=H_{W}$ of Proposition 5.4 can also be written as $H_{x_{1}} * H_{W}=H_{x_{2}} * H_{x_{3}}$. Part (ii) follows from part (i) and Proposition 4.3 .

Now, for a given $W(2)$-quad $W$, there are 15 points contained in $W, 360$ points contained in $\Gamma_{1}(W)$ and 192 points contained in $\Gamma_{2}(W)$. If $x \in \Gamma_{1}(W)$, then every line through $\pi_{W}(x)$ is contained in $H_{W} * H_{x}$. Line "A2" of Table 1, the line distribution mentioned in Table 3 and Corollary 5.5(i) now allow us to identify the two extra hyperplane classes.

Definitions. - A hyperplane of $\mathbb{E}_{3}$ is said to be of Type $A 7$ if it is of the form $H_{W} * H_{x}$, where $W$ is a $W(2)$-quad and $x \in \Gamma_{1}(W)$.

- A hyperplane of $\mathbb{E}_{3}$ is said to be of Type $A 8$ if it is of the form $H_{W} * H_{x}$, where $W$ is a $W(2)$-quad and $x \in \Gamma_{2}(W)$. 


\subsection{Hyperplanes of the form $H_{x} * H_{W}, W$ a $W(2)$-subquadrangle of a $Q(5,2)$-quad}

In this subsection, we give an interpretation to the entries occurring in line "A3" of Table 1. This allows us to identify two extra classes of hyperplanes (A9 and A10).

Proposition 5.6 Let $W$ be a $W(2)$-subquadrangle of a $Q(5,2)$-quad $Q$ and let $x$ be a point of $\mathbb{E}_{3}$.

(i) If $x \in W$, then $H_{x} * H_{W}$ is a hyperplane of Type A3.

(ii) If $x \in Q \backslash W$, then $H_{x} * H_{W}$ is a singular hyperplane whose deepest point belongs to $(Q \backslash W) \cap \Gamma_{2}(x)$.

(iii) If $x \notin H_{W}$, then $H_{x} * H_{W}$ is a hyperplane of Type A6.

(iv) If $x \in \Gamma_{1}(Q) \cap \Gamma_{1}(W)$ such that precisely one of the two $Q(5,2)$-quads through the line $x \pi_{Q}(x)$ intersects $W$ in a line, then $H_{x} * H_{W}$ is a hyperplane of Type A\%.

Proof. Claims (i) and (ii) follow from Lemmas 5.1 and 5.2.

We prove Claim (iii). Suppose that $x \notin H_{W}$. Let $x_{1}$ denote the unique point of $Q$ collinear with $x$. By (ii), $H_{W} * H_{x_{1}}$ is a singular hyperplane whose deepest point $x_{2}$ is contained in $(Q \backslash W) \cap \Gamma_{2}\left(x_{1}\right)$. Now, $H_{x} * H_{W}=H_{x} *\left(H_{x_{1}} * H_{x_{2}}\right)=H_{y} * H_{x_{2}}$, where $y$ denotes the unique point of the line $x x_{1}$ different from $x$ and $x_{1}$. Since $\mathrm{d}\left(y, x_{2}\right)=\mathrm{d}\left(y, x_{1}\right)+\mathrm{d}\left(x_{1}, x_{2}\right)=1+2=3$, $H_{x} * H_{W}=H_{y} * H_{x_{2}}$ is a hyperplane of Type A6.

We prove Claim (iv). Suppose $x$ is a point of $\Gamma_{1}(Q) \cap \Gamma_{1}(W)$ such that precisely one of the two $Q(5,2)$-quads through the line $x \pi_{Q}(x)$ intersects $W$ in a line. Let $Q^{\prime}$ denote the unique $Q(5,2)$-quad through $x \pi_{Q}(x)$ which intersects $Q$ in a line which is not contained in $W$. Let $W_{1}$ denote the other quad through $x \pi_{Q}(x)$ which intersects $Q$ in a line not belonging to $W$. Then $W_{1} \cong W(2)$. Let $y_{1}$ denote an arbitrary point of $\left(W_{1} \cap Q\right) \backslash\left\{\pi_{Q}(x)\right\}$ and put $\left\{y_{1}, x\right\}^{\perp \perp}=\left\{x, y_{1}, y_{3}\right\}$. Now, $H_{y_{1}} * H_{W}$ is a singular hyperplane whose deepest point $y_{2}$ belongs to $(Q \backslash W) \cap \Gamma_{2}\left(y_{1}\right)$. Since $\pi_{Q}(x) \in y_{1}^{\perp} \cap W, \pi_{Q}(x) \in y_{2}^{\perp}$. This implies that $y_{2}$ is contained in $\left(Q^{\prime} \cap Q\right) \backslash\left\{\pi_{Q}(x)\right\}$. Now, there are 3 quads through $\pi_{Q}(x) y_{2}$ which intersect $W_{1}$ in a line. Two of these, namely $Q^{\prime}$ and $Q$, are isomorphic to $Q(5,2)$. The remaining quad $W_{2}:=$ $\left\langle\pi_{Q}(x) y_{2}, \pi_{Q}(x) y_{3}\right\rangle$ is isomorphic to $W(2)$. Put $\left\{y_{2}, y_{3}\right\}^{\perp \perp}=\left\{y_{2}, y_{3}, y_{4}\right\}$ and let $W_{3}$ denote the unique third $W(2)$-quad through the line $W_{1} \cap W_{2}=\pi_{Q}(x) y_{3}$. By Propositions 4.2 and 5.4, $H_{x} * H_{W}=H_{x} * H_{y_{1}} * H_{y_{2}}=\left(H_{x} * H_{y_{1}} * H_{y_{3}}\right) *\left(H_{y_{3}} * H_{y_{4}} * H_{y_{2}}\right) * H_{y_{4}}=H_{W_{1}} * H_{W_{2}} * H_{y_{4}}=H_{W_{3}} * H_{y_{4}}$. This is a hyperplane of Type A7 since $\mathrm{d}\left(y_{4}, W_{3}\right)=1$.

Now, for a given $W(2)$-subquadrangle in a $Q(5,2)$-quad $Q$, there are 15 points in $W, 12$ points in $Q \backslash W, 240$ points in $\Gamma_{2}(W)$ and $180 / 90 / 30$ points $x \in \Gamma_{1}(Q) \cap \Gamma_{1}(W)$ such that precisely $1 / 2 / 0$ of the two $Q(5,2)$-quads through $x \pi_{Q}(x)$ meet $W$ in a line. Observe also that if $x$ is a point of $\Gamma_{1}(Q) \cap \Gamma_{1}(W)$ such that the two $Q(5,2)$-quads through $x \pi_{Q}(x)$ meet $W$ in a line, then $Q_{1}$ and $Q_{2}$ are contained in $H_{x} * H_{W}$. Proposition 5.6, line "A3" of Table 1 and the values of DE mentioned in Table 3 now allow is to identify the two extra hyperplane classes. 
Definitions. - A hyperplane of $\mathbb{E}_{3}$ is said to be of Type $A 9$ if it is of the form $H_{x} * H_{W}$, where $W$ is a $W(2)$-subquadrangle of a $Q(5,2)$-quad $Q$ and $x \in \Gamma_{1}(Q) \cap \Gamma_{1}(W)$ such that each of the two $Q(5,2)$-quads through the line $x \pi_{Q}(x)$ intersects $W$ in a line.

- A hyperplane of $\mathbb{E}_{3}$ is said to be of Type $A 10$ if it is of the from $H_{x} * H_{W}$, where $W$ is a $W(2)$-subquadrangle of a $Q(5,2)$-quad $Q$ and $x \in \Gamma_{1}(Q) \cap \Gamma_{1}(W)$ such that none of the two $Q(5,2)$-quads through the line $x \pi_{Q}(x)$ intersects $W$ in a line.

An alternative construction for the hyperplanes of Type A9 will be given in Proposition 5.8(vii).

\subsection{Hyperplanes of the form $H_{x} * H_{f}, f$ a non-classical valuation of $\mathbb{E}_{3}$}

In this subsection, we give an interpretation to the entries occurring in line "A4" of Table 1. This allows us to identify two extra classes of hyperplanes (A11 and A12).

Proposition 5.7 Let $f$ be a non-classical valuation of $\mathbb{E}_{3}$ and let $x$ be a point with value 0 . Then $H_{x} * H_{f}=H_{f^{\prime}}$ for some non-classical valuation $f^{\prime}$ of $\mathbb{E}_{3}$.

Proof. Let the near hexagon $\mathbb{E}_{3}$ be isometrically embedded into the dual polar space $D H(5,4)$ and let $y$ denote the unique point of $D H(5,4) \backslash \mathbb{E}_{3}$ such that $y^{\perp} \cap \mathbb{E}_{3}=O_{f}$. Notice that $x$ and $y$ are collinear since $x \in O_{f}$. Let $z$ denote the third point on the line $x y$. Let $H_{x}^{\prime}\left(H_{y}^{\prime}\right.$, respectively $\left.H_{z}^{\prime}\right)$ denote the singular hyperplane of $D H(5,4)$ with deepest point $x(y$, respectively $z)$. Then $H_{z}^{\prime}=H_{x}^{\prime} * H_{y}^{\prime}$ and hence $\left(H_{z}^{\prime} \cap \mathbb{E}_{3}\right)=\left(H_{x}^{\prime} \cap \mathbb{E}_{3}\right) *\left(H_{y}^{\prime} \cap \mathbb{E}_{3}\right)$. Now, $H_{x}^{\prime} \cap \mathbb{E}_{3}=H_{x}$ and $H_{y}^{\prime} \cap \mathbb{E}_{3}=H_{f}$. Now, for every point $u$ of $\mathbb{E}_{3}$, define $f^{\prime}(u)=\mathrm{d}(u, z)-1$. Then $f^{\prime}$ is a non-classical valuation of $\mathbb{E}_{3}$ and $H_{z}^{\prime} \cap \mathbb{E}_{3}=H_{f^{\prime}}$. Hence, $H_{x} * H_{f}=H_{f^{\prime}}$.

Now, for a given non-classical valuation $f$ of $\mathbb{E}_{3}$, there are 21 points with value 0,210 points with value 1 and 336 points with value 2 . If $x$ is a point with value 1 and $y \in O_{f} \cap \Gamma_{1}(x)$, then every line through $y$ is contained in $H_{x} * H_{f}$. Proposition 5.7, line "A4" of Table 1 and the line distribution mentioned in Table 3 now allows us to identify the two extra hyperplane classes.

Definitions. - A hyperplane of $\mathbb{E}_{3}$ is said to be of Type $A 11$ if it is of the form $H_{x} * H_{f}$ where $f$ is a non-classical valuation of $\mathbb{E}_{3}$ and $x$ is a point such that $f(x)=1$.

- A hyperplane of $\mathbb{E}_{3}$ is said to be of Type $A 12$ if it is of the form $H_{x} * H_{f}$ where $f$ is a non-classical valuation of $\mathbb{E}_{3}$ and $x$ is a point such that $f(x)=2$.

\subsection{The hyperplanes of the form $H_{W} * H_{x_{1}} * H_{x_{2}}, W$ a $W(2)$-quad and $x_{1} \in W$}

In this subsection, we give an interpretation to the entries occurring in line "A5" of Table 1. This allows us to identify two extra classes of hyperplanes (A13 and A14). Recall that every 
hyperplane of Type A5 is of the form $H_{x} * H_{W}$, where $W$ is a $W(2)$-quad and $x \in W$ (Corollary $5.5(\mathrm{i}))$.

Proposition 5.8 Let $W$ be a $W(2)$-quad of $\mathbb{E}_{3}$ and let $x_{1}, x_{2}$ be points of $\mathbb{E}_{3}$ such that $x_{1} \in W$.

(i) If $x_{1}=x_{2}$, then $H_{W} * H_{x_{1}} * H_{x_{2}}=H_{W}$.

(ii) If $x_{2} \in x_{1}^{\perp} \cap W$, then $H_{W} * H_{x_{1}} * H_{x_{2}}=H_{W} * H_{x_{3}}$, where $x_{3}$ is the unique point of the line $x_{1} x_{2}$ different from $x_{1}$ and $x_{2}$.

(iii) If $x_{2} \in W \backslash x_{1}^{\perp}$ and $\left\{x_{1}, x_{2}\right\}^{\perp \perp}=\left\{x_{1}, x_{2}, x_{3}\right\}$, then $H_{W} * H_{x_{1}} * H_{x_{2}}=H_{x_{3}}$.

(iv) If $x_{2} \in x_{1}^{\perp} \backslash W$, then $H_{W} * H_{x_{1}} * H_{x_{2}}=H_{W} * H_{x_{3}}$, where $x_{3}$ is the unique point on the line $x_{1} x_{2}$ different from $x_{1}$ and $x_{2}$.

(v) If $x_{2} \in \Gamma_{1}(W) \cap \Gamma_{3}\left(x_{1}\right)$, then $H_{W} * H_{x_{1}} * H_{x_{2}}$ is a hyperplane of Type $A 6$.

(vi) If $x_{2} \in \Gamma_{1}(W) \cap \Gamma_{2}\left(x_{1}\right)$ such that $\left\langle x_{1}, x_{2}\right\rangle$ is a W(2)-quad, then $H_{W} * H_{x_{1}} * H_{x_{2}}$ is a hyperplane of Type $A \%$.

(vii) If $x_{2} \in \Gamma_{1}(W) \cap \Gamma_{2}\left(x_{1}\right)$ such that $\left\langle x_{1}, x_{2}\right\rangle$ is a $Q(5,2)$-quad, then $H_{W} * H_{x_{1}} * H_{x_{2}}$ is a hyperplane of Type A9.

Proof. In case (i), $H_{W} * H_{x_{1}} * H_{x_{2}}=H_{W} *\left(H_{x_{1}} * H_{x_{2}}\right)=H_{W} * \mathbb{E}_{3}=H_{W}$. In cases (ii) and (iv), $H_{W} * H_{x_{1}} * H_{x_{2}}=H_{W} *\left(H_{x_{1}} * H_{x_{2}}\right)=H_{W} * H_{x_{3}}$. In case (iii), $H_{W} * H_{x_{1}} * H_{x_{2}}=$ $\left(H_{x_{1}} * H_{x_{2}} * H_{x_{3}}\right) * H_{x_{1}} * H_{x_{2}}=H_{x_{3}}$ (cf. Proposition 5.4).

Suppose now that $x_{2} \in \Gamma_{1}(W) \cap \Gamma_{3}\left(x_{1}\right)$. Let $x_{2}^{\prime}$ denote the unique point of $W$ collinear with $x_{2}$, let $x_{2}^{\prime \prime}$ denote the unique third point on the line $x_{2} x_{2}^{\prime}$ and put $\left\{x_{1}, x_{2}^{\prime}\right\}^{\perp \perp}=\left\{x_{1}, x_{2}^{\prime}, x_{3}\right\}$. Then $H_{W} * H_{x_{1}} * H_{x_{2}}=\left(H_{x_{1}} * H_{x_{3}} * H_{x_{2}^{\prime}}\right) * H_{x_{1}} * H_{x_{2}}=H_{x_{3}} * H_{x_{2}^{\prime \prime}}$. This is a hyperplane of Type A6 since $\mathrm{d}\left(x_{3}, x_{2}^{\prime \prime}\right)=\mathrm{d}\left(x_{3}, x_{2}^{\prime}\right)+\mathrm{d}\left(x_{2}^{\prime}, x_{2}^{\prime \prime}\right)=2+1=3$.

Suppose now that $x_{2} \in \Gamma_{1}(W) \cap \Gamma_{2}\left(x_{1}\right)$ such that $\left\langle x_{1}, x_{2}\right\rangle$ is a $W(2)$-quad. Let $x_{2}^{\prime}$ denote the unique point of $W$ collinear with $x_{2}$, let $x_{3} \in \Gamma_{2}\left(x_{1}\right) \cap \Gamma_{1}\left(x_{2}^{\prime}\right) \cap W$ and put $\left\{x_{1}, x_{3}\right\}^{\perp \perp}=$ $\left\{x_{1}, x_{3}, x_{4}\right\}$. Then $H_{W} * H_{x_{1}} * H_{x_{2}}=\left(H_{x_{1}} * H_{x_{3}} * H_{x_{4}}\right) * H_{x_{1}} * H_{x_{2}}=\left(H_{x_{2}} * H_{x_{3}}\right) * H_{x_{4}}$. The two $Q(5,2)$-quads through the line $x_{2} x_{2}^{\prime}$ intersect $W$ in lines and hence coincide with $\left\langle x_{2}, x_{3}\right\rangle$ and $\left\langle x_{2}, x_{4}\right\rangle$. It follows that $H_{x_{2}} * H_{x_{3}}=H_{W^{\prime}}$, where $W^{\prime}$ is a $W(2)$-subquadrangle of $\left\langle x_{2}, x_{3}\right\rangle$ containing the point $x_{2}^{\prime} \in x_{2}^{\perp} \cap x_{3}^{\perp}$, but not the lines $x_{2}^{\prime} x_{2}$ and $x_{2}^{\prime} x_{3}$. Since $\mathrm{d}\left(x_{4}, W^{\prime}\right)=$ $\mathrm{d}\left(x_{4}, x_{2}^{\prime}\right)=1,\left\langle x_{4}, x_{2}\right\rangle \cong Q(5,2)$ and $\left\langle x_{4}, x_{3}\right\rangle \cong W(2), H_{W} * H_{x_{1}} * H_{x_{2}}=H_{W^{\prime}} * H_{x_{4}}$ is a hyperplane of Type A7 by Proposition 5.6 (iv).

Suppose now that $x_{2} \in \Gamma_{1}(W) \cap \Gamma_{2}\left(x_{1}\right)$ such that $\left\langle x_{1}, x_{2}\right\rangle$ is a $Q(5,2)$-quad. Let $x_{2}^{\prime}$ denote the unique point of $W$ collinear with $x_{2}$, let $x_{3} \in \Gamma_{2}\left(x_{1}\right) \cap \Gamma_{1}\left(x_{2}^{\prime}\right) \cap W$ such that $\left\langle x_{2}, x_{3}\right\rangle \cong Q(5,2)$ and put $\left\{x_{1}, x_{3}\right\}^{\perp \perp}=\left\{x_{1}, x_{3}, x_{4}\right\}$. Then $H_{W} * H_{x_{1}} * H_{x_{2}}=\left(H_{x_{1}} * H_{x_{3}} * H_{x_{4}}\right) * H_{x_{1}} * H_{x_{2}}=$ $\left(H_{x_{2}} * H_{x_{3}}\right) * H_{x_{4}}=H_{W^{\prime}} * H_{x_{4}}$, where $W^{\prime}$ is a $W(2)$-subquadrangle of $\left\langle x_{2}, x_{3}\right\rangle$ containing the point $x_{2}^{\prime} \in x_{2}^{\perp} \cap x_{3}^{\perp}$, but not the lines $x_{2}^{\prime} x_{2}$ and $x_{2}^{\prime} x_{3}$. Since $\mathrm{d}\left(x_{4}, W^{\prime}\right)=\mathrm{d}\left(x_{4}, x_{2}^{\prime}\right)=1$ and $\left\langle x_{4}, x_{2}\right\rangle \cong W(2) \cong\left\langle x_{4}, x_{3}\right\rangle, H_{W} * H_{x_{1}} * H_{x_{2}}=H_{W^{\prime}} * H_{x_{4}}$ is a hyperplane of Type A9.

Now, for every $W(2)$-quad $W$ and every point $x_{1} \in W$, there is 1 point coinciding with $x_{1}, 6$ points in $x_{1}^{\perp} \cap W, 8$ points in $W \backslash x_{1}^{\perp}, 24$ points in $x_{1}^{\perp} \backslash W, 192$ points in $\Gamma_{1}(W) \cap \Gamma_{3}\left(x_{1}\right), 48$ points $x_{2} \in \Gamma_{1}(W) \cap \Gamma_{2}\left(x_{1}\right)$ such that $\left\langle x_{1}, x_{2}\right\rangle$ is a $W(2)$-quad, 96 points $x_{2}^{\prime} \in \Gamma_{1}(W) \cap \Gamma_{2}\left(x_{1}\right)$ such that $\left\langle x_{1}, x_{2}^{\prime}\right\rangle$ is a $Q(5,2)$-quad, 64 points in $\Gamma_{2}\left(x_{1}\right) \cap \Gamma_{2}(W)$ and 128 points in $\Gamma_{3}\left(x_{1}\right) \cap \Gamma_{2}(W)$. 
If $x_{2} \in \Gamma_{3}\left(x_{1}\right) \cap \Gamma_{2}(W)$ and $Q$ is a $Q(5,2)$-quad through $x_{2}$ (necessarily disjoint from $W$ ), then the unique point $y$ of $Q$ collinear with $x_{1}$ belongs to $\Gamma_{1}\left(x_{1}\right) \cap \Gamma_{1}(W) \cap \Gamma_{2}\left(x_{2}\right)$ and there are precisely 5 lines through $y$ which are completely contained in $H_{x_{1}} * H_{W} * H_{x_{2}}$. Proposition 5.8, line "A5" of Table 1 and the line distribution mentioned in Table 3 now allow us to identify the two extra hyperplane classes.

Definitions. - A hyperplane of $\mathbb{E}_{3}$ is said to be of Type $A 13$ if it is of the form $H_{W} * H_{x_{1}} * H_{x_{2}}$, where $W$ is a $W(2)$-quad, $x_{1} \in W$ and $x_{2} \in \Gamma_{2}\left(x_{1}\right) \cap \Gamma_{2}(W)$.

- A hyperplane of $\mathbb{E}_{3}$ is said to be of Type $A 14$ if it is of the form $H_{W} * H_{x_{1}} * H_{x_{2}}$, where $W$ is a $W(2)$-quad, $x_{1} \in W$ and $x_{2} \in \Gamma_{3}\left(x_{1}\right) \cap \Gamma_{2}(W)$.

\subsection{The remaining hyperplanes of Type A}

We still need to define three extra classes of Type A hyperplanes. In Section 4, we studied the possible configurations of two distinct $W(2)$-quads $W_{1}$ and $W_{2}$, and determined which kind of hyperplane $H_{W_{1}} * H_{W_{2}}$ is. There was however one case which we did not consider. If $W_{1}$ and $W_{2}$ are two $W(2)$-quads satisfying condition (5) of Proposition 4.1, then by counting we find that $\left|H_{W_{1}} \cap H_{W_{2}}\right|=243$. Hence, $\left|H_{W_{1}} * H_{W_{2}}\right|=567-\left|H_{W_{1}}\right|-\left|H_{W_{2}}\right|+2 \cdot\left|H_{W_{1}} \cap H_{W_{2}}\right|=303$. Table 3 now allows us to identify an extra class of hyperplanes.

Definition. - A hyperplane of $\mathbb{E}_{3}$ is said to be of Type $A 15$ if it is of the form $H_{W_{1}} * H_{W_{2}}$, where $W_{1}$ and $W_{2}$ are two $W(2)$-quads satisfying $W_{1} \cap W_{2}=\emptyset, W_{2} \cap \Gamma_{1}\left(W_{1}\right)$ is a $(3 \times 3)$-subgrid of $W_{2}$ and $W_{1} \cap \Gamma_{1}\left(W_{2}\right)$ is a $(3 \times 3)$-subgrid of $W_{1}$.

In order to define the hyperplanes of Type A16 and A17, we need to define a certain configuration of two $W(2)$-subquadrangles. Let $Q$ and $Q^{\prime}$ be two disjoint $Q(5,2)$-quads of $\mathbb{E}_{3}$. Let $\left\{G_{1}, G_{2}, G_{3}\right\}$ be the partition of $Q$ in three $(3 \times 3)$-subgrids such that for every line $L$ of $G_{i}$, $i \in\{1,2,3\}$, the quad $\left\langle L, \pi_{Q^{\prime}}(L)\right\rangle$ is isomorphic to $Q(5,2)$. Put $G_{i}^{\prime}:=\pi_{Q^{\prime}}\left(G_{i}\right), i \in\{1,2,3\}$. Let $W$ denote a $W(2)$-subquadrangle of $Q$ through $G_{1}$, let $W^{\prime}$ be a $W(2)$-subquadrangle of $Q^{\prime}$ through $G_{1}^{\prime}$ such that $W^{\prime} \neq \pi_{Q^{\prime}}(W)$ and let $x$ be an arbitrary point of $G_{1}$. By counting, we find that $\left|H_{W} \cap H_{W^{\prime}}\right|=219$. (One can consider all the quads which meet $Q$ and $Q^{\prime}$ in lines. These determine a partition of the points outside $Q \cup Q^{\prime} \cup \mathcal{R}_{Q}\left(Q^{\prime}\right)$.) Hence, $\left|H_{W} * H_{W^{\prime}}\right|=567-\left|H_{W}\right|-\left|H_{W^{\prime}}\right|+2 \cdot\left|H_{W} \cap H_{W^{\prime}}\right|=351$. Table 3 now allows us to identify an extra class of hyperplanes.

Definition. - A hyperplane of $\mathbb{E}_{3}$ is said to be of Type $A 16$ if it is of the form $H_{W} * H_{W^{\prime}}$, where $W$ and $W^{\prime}$ are two $W(2)$-subquadrangles which are obtained in the way described above. In Section 6, we will discuss the structure of the hyperplanes of Type A16.

The two $Q(5,2)$-quads through $x$ which meet $G_{1}$ and $G_{1}^{\prime}$ in lines are contained in $H_{W^{*}} * H_{W^{\prime}} * H_{x}$. Line "A16" of Table 1 and the values of DE mentioned in Table 3 now allow us to identify the last class of Type A hyperplanes. 
Definition. • A hyperplane of $\mathbb{E}_{3}$ is said to be of Type Type $A 17$ if it is of the form $H_{W^{*}} * H_{W^{\prime}} * H_{x}$ where $W$ and $W^{\prime}$ are two $W(2)$-subquadrangles and $x$ is a point obtained in the way described above.

\subsection{The hyperplanes of Type B}

The aim of this subsection is to give explicit constructions for the Type B hyperplanes.

Lemma 5.9 Let the near hexagon $\mathbb{G}_{3}$ be isometrically embedded as a hyperplane into the near hexagon $\mathbb{E}_{3}$. Then every quad of $\mathbb{E}_{3}$ intersects $\mathbb{G}_{3}$ in a quad of $\mathbb{G}_{3}$.

Proof. If $Q$ is a quad of $\mathbb{G}_{3}$, then there exists a unique quad $\bar{Q}$ in $\mathbb{E}_{3}$ such that $Q=\bar{Q} \cap \mathbb{G}_{3}$. The quad $\bar{Q}$ is the smallest convex subspace of $\mathbb{E}_{3}$ containing $Q$. The lemma now follows from the fact that both the near hexagons $\mathbb{G}_{3}$ and $\mathbb{E}_{3}$ contain precisely 693 quads.

So, with respect to an isometric embedding of $\mathbb{G}_{3}$ into $\mathbb{E}_{3}$, there are two types of $Q(5,2)$-quads in $\mathbb{E}_{3}$. There are $Q(5,2)$-quads which are contained in $\mathbb{G}_{3}$ and there are $Q(5,2)$-quads which intersect $\mathbb{G}_{3}$ in a $W(2)$-quad of $\mathbb{G}_{3}$. Similarly, there are two types of $W(2)$-quads in $\mathbb{E}_{3}$. There are $W(2)$-quads which are contained in $\mathbb{G}_{3}$ and there are $W(2)$-quad which intersect $\mathbb{G}_{3}$ in a grid-quad of $\mathbb{G}_{3}$.

Recall that a hyperplane $H$ of $\mathbb{E}_{3}$ is said to be of Type B1 if it carries the structure of a near hexagon isomorphic to $\mathbb{G}_{3}$. A line of $\mathbb{E}_{3}$ contained in a Type B1 hyperplane $H$ is said to be a special line (respectively an ordinary line) of $H$ if it is a special (respectively ordinary) line of the $\mathbb{G}_{3}$ near hexagon associated with $H$. (Recall the definitions given in Section 2.) We now give explicit constructions of the remaining Type B hyperplanes occurring in Table 2.

Definitions. - A hyperplane of $\mathbb{E}_{3}$ is said to be of Type B2 if it is of the form $H * H_{x}$ where $H$ is a hyperplane of Type B1 and where $x$ is a point of $H$.

- A hyperplane of $\mathbb{E}_{3}$ is said to be of Type $B 3$ if it is of the form $H * H_{x}$ where $H$ is a hyperplane of Type $\mathrm{B} 1$ and $x$ is a point not belonging to $H$.

- A hyperplane of $\mathbb{E}_{3}$ is said to be of Type $B 4$ if it is of the form $H * H_{W}$ where $H$ is a hyperplane of Type $\mathrm{B} 1$ and $W$ is a $W(2)$-quad contained in $H$.

- A hyperplane of $\mathbb{E}_{3}$ is said to be of Type $B 5$ if it is of the form $H * H_{W}$ where $H$ is a hyperplane of Type B1 and $W$ is a $W(2)$-quad not contained in $H$.

- A hyperplane of $\mathbb{E}_{3}$ is said to be of Type B6 if it is of the from $H * H_{W} * H_{x}$, where $H$ is a hyperplane of Type B1, $W$ is a $W(2)$-quad contained in $H$ and $x \in W$.

- A hyperplane of $\mathbb{E}_{3}$ is said to be of Type B7 if it is of the form $H * H_{W} * H_{x}$, where $H$ is a hyperplane of Type B1, $W$ is a $W(2)$-quad contained in $H$ and $x \in \Gamma_{1}(W) \cap H$ such that the unique line through $x$ meeting $W$ is an ordinary line of $H$.

- A hyperplane of $\mathbb{E}_{3}$ is said to be of Type B8 if it is of the form $H * H_{W} * H_{x}$, where $H$ is a hyperplane of Type B1, $W$ is a $W(2)$-quad contained in $H$ and $x \in \Gamma_{2}(W) \cap H$. 
- A hyperplane of $\mathbb{E}_{3}$ is said to be of Type $B 9$ if it is of the form $H * H_{W} * H_{x}$, where $H$ is a hyperplane of Type B1, $W$ is a $W(2)$-quad not contained in $H$ and $x$ is one of the 9 points of $W \cap H$.

- A hyperplane of $\mathbb{E}_{3}$ is said to be of Type B10 if it is of the form $H * H_{W} * H_{x}$, where $H$ is a hyperplane of Type B1, $W$ is a $W(2)$-quad not contained in $H$ and $x$ is one of the 6 points in $W \backslash H$.

We have verified with the aid of a computer that the hyperplanes of Type B $i(i \in\{2, \ldots, 10\})$ as defined above agree with those which occur in Tables 2 and 3. It is also possible (with some effort) to derive this only from the information provided by these tables. Let us do this for the hyperplanes of Type B6 and B7, since the reasoning will also provide information on the hyperplanes of the form $H * H_{W} * H_{x}$, where $H$ is a Type B1 hyperplane, $W$ a $W(2)$-quad contained in $H$ and $x \in \Gamma_{1}(W) \cap H$ such that $x \pi_{W}(x)$ is a special line.

Suppose $H$ is a Type B1 hyperplane, $W$ a $W(2)$-quad contained in $H$ and $y$ a point. Recall that every $Q(5,2)$-quad which meets $W$ intersects $W$ in a line. Assume that we already know that $H * H_{W}$ is a Type B4 hyperplane (as occurring in Tables 2 and 3). By Table $2, H * H_{W} * H_{y}$ is of Type B6, B7 or B8. If $y$ is one of the 15 points of $W$, then $H * H_{W} * H_{y}$ is of type B6, since the three $Q(5,2)$-quads through $y$ contained in $H$ are deep with respect to $H * H_{W} * H_{y}$ (observe the values of DE in Table 3). If $y$ is one of the 90 points of $\Gamma_{1}(W) \cap H$ such that the line $y \pi_{W}(y)$ is special, then $H * H_{W} * H_{y}$ is of Type B6, since the two $Q(5,2)$-quads through $y \pi_{W}(y)$ are deep. So, we have located all $105=90+15$ points $y$ for which $H * H_{W} * H_{y}$ is of Type B6. Now, if $y$ is a point of $\Gamma_{1}(W) \cap H$ for which the line $y \pi_{W}(y)$ is ordinary, then the unique $Q(5,2)$-quad through $y \pi_{W}(y)$ contained in $H$ is deep and hence $H * H_{W} * H_{y}$ must be of Type B7 by Table 3.

\section{The structure of the hyperplanes of Type A16}

Besides the basic hyperplane classes, there is one additional class whose hyperplanes seem to have a "nice structure", namely the Type A16 hyperplanes. Indeed, looking at Table 3, we observe that every point of such a hyperplane $H$ is incident with either 9 or 11 lines which are contained in $H$. The aim of this section is to discuss the structure of the hyperplanes of Type A16 by means of an alternative construction we will give for these hyperplanes.

Let $Q_{1}$ and $Q_{2}$ be two disjoint $Q(5,2)$-quads of $\mathbb{E}_{3}$ and put $Q_{3}:=\mathcal{R}_{Q_{1}}\left(Q_{2}\right)=\mathcal{R}_{Q_{2}}\left(Q_{1}\right)$. Let $\mathcal{V}$ denote the set of all $18 Q(5,2)$-quads of $\mathbb{E}_{3}$ which intersect each of $Q_{1}, Q_{2}, Q_{3}$ in a line. The quad $Q_{1}$ can be partitioned into 3 grids $G_{1}, G_{2}$ and $G_{3}$ such that if $L$ is a line of $G_{i}, i \in\{1,2,3\}$, then $\left\langle L, \pi_{Q_{2}}(L)\right\rangle \in \mathcal{V}$. Let $\mathcal{W}$ denote the set of all 18 lines of the form $Q_{1} \cap Q$ where $Q \in \mathcal{V}$. Let $S$ be a regular spread of $Q_{1}$ such that every line of $S$ is contained in $\mathcal{W}$. Let $R_{1}, R_{2}, \ldots, R_{9}$ be the $9 Q(5,2)$-quads of $\mathcal{V}$ such that $R_{i} \cap Q_{1} \in S$ for every $i \in\{1, \ldots, 9\}$. Without loss of generality, we may suppose that (i) $R_{1} \cap Q_{1}, R_{2} \cap Q_{1}, R_{3} \cap Q_{1}$ are lines of $G_{1}$; (ii) $R_{4} \cap Q_{1}, R_{5} \cap Q_{1}$, $R_{6} \cap Q_{1}$ are lines of $G_{2}$; (iii) $R_{7} \cap Q_{1}, R_{8} \cap Q_{1}, R_{9} \cap Q_{1}$ are lines of $G_{3}$. The set $Q_{1} \cup Q_{2} \cup Q_{3}$ 
intersects $R_{1}$ in a $(3 \times 3)$-grid $G_{1}^{\prime}$. Let $G_{2}^{\prime}$ and $G_{3}^{\prime}$ denote the $(3 \times 3)$-subgrids of $R_{1}$ such that $\left\{G_{1}^{\prime}, G_{2}^{\prime}, G_{3}^{\prime}\right\}$ is a partition of $R_{1}$ into 3 subgrids. Now, let $x$ denote an arbitrary point of $G_{2}^{\prime}$. Let $L_{1}$ denote the unique line through $x$ meeting $R_{2}$ and $R_{3}$ and let $L_{2}$ denote the unique line through $x$ meeting $R_{4}$. The quad $\left\langle L_{1}, L_{2}\right\rangle$ intersects each of the quads $R_{1}, R_{2}, R_{3}, R_{4}$ in a line since these quads are isomorphic to $Q(5,2)$. There are two possibilities for the line $\left\langle L_{1}, L_{2}\right\rangle \cap R_{1}$. Either this line is contained in $G_{2}^{\prime}$ or this line meets $G_{1}^{\prime}$. Suppose the latter case occurs. Then without loss of generality, we may suppose that $\left\langle L_{1}, L_{2}\right\rangle$ meets $Q_{1}$ (necessarily in a line). Then $\pi_{Q_{1}}\left(\left\langle L_{1}, L_{2}\right\rangle\right)$ is a line. But this is impossible since $\pi_{Q_{1}}\left(\left\langle L_{1}, L_{2}\right\rangle\right)$ contains the point $\pi_{Q_{1}}\left(L_{1} \cap R_{1}\right)$ of $R_{1} \cap Q_{1}$, the point $\pi_{Q_{1}}\left(L_{1} \cap R_{2}\right)$ of $R_{2} \cap Q_{1}$, the point $\pi_{Q_{1}}\left(L_{1} \cap R_{3}\right)$ of $R_{3} \cap Q_{1}$ and the point $\pi_{Q_{1}}\left(L_{2} \cap R_{4}\right)$ of $R_{4} \cap Q_{1}$. Hence, $\left\langle L_{1}, L_{2}\right\rangle$ is disjoint from $Q_{1} \cup Q_{2} \cup Q_{3}$ and $\left\langle L_{1}, L_{2}\right\rangle \cap R_{1}$ is a line belonging to $G_{2}^{\prime}$. Put $Q_{4}:=\left\langle L_{1}, L_{2}\right\rangle$. Then $\pi_{Q_{1}}\left(Q_{4}\right)$ contains the lines $\pi_{Q_{1}}\left(Q_{4} \cap R_{1}\right)=R_{1} \cap Q_{1}$, $\pi_{Q_{1}}\left(Q_{4} \cap R_{2}\right)=R_{2} \cap Q_{1}, \pi_{Q_{1}}\left(Q_{4} \cap R_{3}\right)=R_{3} \cap Q_{1}$ and $\pi_{Q_{1}}\left(Q_{4} \cap R_{4}\right)=R_{4} \cap Q_{1}$. The smallest subspace of $Q_{1}$ containing $\left(R_{1} \cap Q_{1}\right) \cup\left(R_{2} \cap Q_{1}\right) \cup\left(R_{3} \cap Q_{1}\right) \cup\left(R_{4} \cap Q_{1}\right)=G_{1} \cup\left(R_{4} \cap Q_{1}\right)$ coincides with $Q_{1}$. Hence, $\pi_{Q_{1}}\left(Q_{4}\right)=Q_{1}$ and $Q_{4}$ is a $Q(5,2)$-quad. Since $Q_{4}$ intersects each of $R_{1}, R_{2}, R_{3}$, $R_{4}$ in a line, it also intersects each of $\mathcal{R}_{R_{4}}\left(R_{1}\right), \mathcal{R}_{R_{4}}\left(R_{2}\right), \mathcal{R}_{R_{4}}\left(R_{3}\right)$, i.e. each of $R_{7}, R_{8}, R_{9}$, in a line. In a similar way one shows that $Q_{4}$ intersects each of $R_{5}, R_{6}$ in a line. Now, let $S^{\prime}$ denote the unique regular spread of $R_{1}$ containing the lines $Q_{1} \cap R_{1}, Q_{2} \cap R_{1}, Q_{3} \cap R_{1}, Q_{4} \cap R_{1}$ and let $Q_{5}, Q_{6}, Q_{7}, Q_{8}, Q_{9}$ denote the $Q(5,2)$-quads of $\mathbb{E}_{3}$ such that $\left\{Q_{i} \mid 1 \leq i \leq 9\right\}$ are all the $9 Q(5,2)$ quads of $\mathbb{E}_{3}$ which intersects $R_{1}$ in a line of $S^{\prime}$. Without loss of generality, we may suppose that $Q_{5} \cap R_{1} \subseteq G_{2}^{\prime}, Q_{6} \cap R_{1} \subseteq G_{2}^{\prime}, Q_{7} \cap R_{1} \subseteq G_{3}^{\prime}, Q_{8} \cap R_{1} \subseteq G_{3}^{\prime}, Q_{9} \cap R \subseteq G_{3}^{\prime}$. Since $Q_{1}, Q_{2}, Q_{3}, Q_{4}$ are $Q(5,2)$-quads which intersect each quad $R_{i}, i \in\{1,2, \ldots, 9\}$, in a line, also $\mathcal{R}_{Q_{1}}\left(Q_{4}\right), \mathcal{R}_{Q_{2}}\left(Q_{4}\right)$ and $\mathcal{R}_{Q_{3}}\left(Q_{4}\right)$ intersect each quad $R_{i}, i \in\{1,2, \ldots, 9\}$, in a line. In other words, $Q_{7}, Q_{8}, Q_{9}$ intersect each quad $R_{i}, i \in\{1,2, \ldots, 9\}$ in a line. With a similar reasoning, one shows that also $Q_{5}, Q_{6} \in\left\{\mathcal{R}_{Q_{1}}\left(Q_{7}\right), \mathcal{R}_{Q_{1}}\left(Q_{8}\right), \mathcal{R}_{Q_{1}}\left(Q_{9}\right)\right\}$ intersect each quad $R_{i}, i \in\{1,2, \ldots, 9\}$, in a line. It is now easily seen that the set $X:=Q_{1} \cup Q_{2} \cup \cdots \cup Q_{9}=R_{1} \cup R_{2} \cup \cdots \cup R_{9}$ carries the structure of a $Q(5,2) \otimes Q(5,2)$ near hexagon. Put $S^{*}:=\left\{Q_{i} \cap R_{j} \mid 1 \leq i, j \leq 9\right\}$.

Now, let $\mathbb{E}_{3}$ be isometrically embedded into the dual polar space $D H(5,4)$. By De Bruyn and Pralle [17, Section 4.6], there are 3 hyperplanes of $D H(5,4)$ which contain $X$ and which arise from the Grassmann-embedding of $D H(5,4)$. If $H$ is one of these three hyperplanes, then $H$ satisfies the following properties:

- Through every point $x \in H \backslash X$, there are 9 lines which are contained in $H$. These are precisely the 9 lines through $x$ meeting one of the quads $Q_{1}, Q_{2}, \ldots, Q_{9}$, or equivalently, the 9 lines through $x$ meeting one of the quads $R_{1}, R_{2}, \ldots, R_{9}$.

- If $Q$ is a $Q(5,2)$-quad through one of the lines of $S^{*}$ such that $Q \notin\left\{Q_{1}, Q_{2}, \ldots, Q_{9}, R_{1}, R_{2}\right.$, $\left.\ldots, R_{9}\right\}$, then $Q$ is singular with respect to $H$.

- Through every point $x \in X$, there are 13 lines which are contained in $H$.

It is now clear that the hyperplane $H \cap \mathbb{E}_{3}$ of $\mathbb{E}_{3}$ satisfies the following properties:

- Through every point $x \in H \backslash X$, there are 9 lines which are contained in $H$. These are precisely the 9 lines through $x$ meeting one of the quads $Q_{1}, Q_{2}, \ldots, Q_{9}$, or equivalently, the 9 lines through $x$ meeting one of the quads $R_{1}, R_{2}, \ldots, R_{9}$. 
- Let $Q$ be a $Q(5,2)$-quad of $D H(5,4)$ through one of the lines of $S^{*}$ such that $Q \notin$ $\left\{Q_{1}, Q_{2}, \ldots, Q_{9}, R_{1}, R_{2}, \ldots, R_{9}\right\}$. Then $Q$ intersects $\mathbb{E}_{3}$ in a $W(2)$-quad of $\mathbb{E}_{3}$. (Notice that there are already two $Q(5,2)$-quads of the set $\left\{Q_{1}, \ldots, Q_{9}, R_{1}, \ldots, R_{9}\right\}$ through that line of $S^{*}$.) The $W(2)$-quad $Q \cap \mathbb{E}_{3}$ of $\mathbb{E}_{3}$ is singular with respect to the hyperplane $H \cap \mathbb{E}_{3}$ of $\mathbb{E}_{3}$.

- Through every point $x \in X$, there are 11 lines which are contained in $H$.

By Table 3, the hyperplane $H \cap \mathbb{E}_{3}$ of $\mathbb{E}_{3}$ must be a hyperplane of Type A16. If $x$ is an arbitrary point of $X$, then $H_{x} *\left(H \cap \mathbb{E}_{3}\right)$ is a hyperplane of Type A17, since the two $Q(5,2)$-quads through $x$ contained in $X$ are deep with respect to $H_{x} *\left(H \cap \mathbb{E}_{3}\right)$. (Recall line "A16" of Table 1 and the values of DE in Table 3.) So, we have located all $243=|X|$ points $x$ for which $H_{x} *\left(H \cap \mathbb{E}_{3}\right)$ is a hyperplane of Type A17.

\section{Acknowledgment}

The first author is a Postdoctoral Fellow of the Research Foundation - Flanders (Belgium)

\section{References}

[1] M. Aschbacher. Flag structures on Tits geometries. Geom. Dedicata 14 (1983), 21-32.

[2] M. K. Bardoe. On the universal embedding of the near-hexagon for $U_{4}(3)$. Geom. Dedicata 56 (1995), 7-17.

[3] A. E. Brouwer, A. M. Cohen, J. I. Hall and H. A. Wilbrink. Near polygons and Fischer spaces. Geom. Dedicata 49 (1994), 349-368.

[4] A. E. Brouwer, H. Cuypers and E. W. Lambeck. The hyperplanes of the $M_{24}$ near polygon. Graphs Combin. 18 (2002), 415-420.

[5] A. E. Brouwer and H. A. Wilbrink. The structure of near polygons with quads. Geom. Dedicata 14 (1983), 145-176.

[6] P. J. Cameron. Dual polar spaces. Geom. Dedicata 12 (1982), 75-85.

[7] I. Cardinali, B. De Bruyn and A. Pasini. Minimal full polarized embeddings of dual polar spaces. J. Algebraic Combin. 25 (2007), 7-23.

[8] B. N. Cooperstein. On the generation of dual polar spaces of unitary type over finite fields. European J. Combin. 18 (1997), 849-856.

[9] B. De Bruyn. On near hexagons and spreads of generalized quadrangles. J. Algebraic Combin. 11 (2000), 211-226. 
[10] B. De Bruyn. The generating rank of the $U_{4}(3)$ near hexagon. Discrete Math. 307 (2007), 2900-2905.

[11] B. De Bruyn. The universal embedding of the near polygon $\mathbb{G}_{n}$. Electron. J. Comb. 14. Research paper R39, 12p. (2007).

[12] B. De Bruyn. On the Grassmann-embeddings of the Hermitian dual polar spaces. Linear Multilinear Algebra 56 (2008), 665-677.

[13] B. De Bruyn. Two new classes of hyperplanes of the dual polar space $D H(2 n-1,4)$ not arising from the Grassmann-embedding. Linear Algebra Appl. 429 (2008), 2030-2045.

[14] B. De Bruyn. The hyperplanes of $D W\left(5,2^{h}\right)$ which arise from embedding. Discrete Math. 309 (2009), 304-321.

[15] B. De Bruyn. Dual embeddings of dense near polygons. Ars Combinatoria, to appear.

[16] B. De Bruyn and H. Pralle. The exceptional hyperplanes of $D H(5,4)$. European J. Combin. 28 (2007), 1412-1418.

[17] B. De Bruyn and H. Pralle. The hyperplanes of DH(5, $\left.q^{2}\right)$. Forum Math. 20 (2008), 239264.

[18] B. De Bruyn and H. Pralle. The hyperplanes of the near hexagon on the 2-factors of the complete graph $K_{8}$. Discrete Math. 308 (2008), 5656-5671.

[19] B. De Bruyn and S. Shpectorov. The hyperplanes of the near hexagon related to the extended ternary Golay code. In preparation.

[20] B. De Bruyn and P. Vandecasteele. Valuations of near polygons. Glasg. Math. J. 47 (2005), 347-361.

[21] B. De Bruyn and P. Vandecasteele. The distance-2-sets of the slim dense near hexagons. Ann. Comb. 10 (2006), 193-210.

[22] The GAP Group, GAP - Groups, Algorithms, and Programming, Version 4.4.12; 2008. (http://www.gap-system.org)

[23] W. M. Kantor. Some geometries that are almost buildings. European J. Combin. 2 (1981), 239-247.

[24] P. Li. On the universal embedding of the $U_{2 n}(2)$ dual polar space. J. Combin. Theory Ser. A 98 (2002), 235-252.

[25] A. Pasini and S. Shpectorov. Uniform hyperplanes of finite dual polar spaces of rank 3. J. Combin. Theory Ser. A 94 (2001), 276-288. 
[26] S. E. Payne and J. A. Thas. Finite Generalized Quadrangles. Research Notes in Mathematics 110. Pitman, Boston, 1984.

[27] H. Pralle. The hyperplanes of DW(5,2). Experiment. Math. 14 (2005), 373-384.

[28] M. A. Ronan. Embeddings and hyperplanes of discrete geometries. European J. Combin. 8 (1987), 179-185.

[29] M. A. Ronan and S. D. Smith. 2-local geometries for some sporadic groups. The Santa Cruz Conference on Finite Groups (Univ. California, Santa Cruz, Calif., 1979). Proc. Sympos. Pure Math. 37 (1980), 283-289.

[30] E. E. Shult. On Veldkamp lines. Bull. Belg. Math. Soc. Simon Stevin 4 (1997), 299-316.

[31] S. Yoshiara. Embeddings of flag-transitive classical locally polar geometries of rank 3. Geom. Dedicata 43 (1992), 121-165.

\section{Addresses:}

Bart De Bruyn, Department of Pure Mathematics and Computer Algebra, Ghent University, Krijgslaan 281 (S22), B-9000 Gent, Belgium, bdb@cage.ugent.be

Sergey Shpectorov, School of Mathematics, University of Birmingham, Edgbaston, Birmingham B15 2TT, United Kingdom, s.shpectorov@bham.ac.uk 